\title{
ON THE PERFECT ORDERABILITY OF UNIONS OF TWO GRAPHS
}

\author{
A Thesis \\ Submitted to the Faculty of Graduate Studies and Research \\ In Partial Fulfillment of the Requirements \\ FOR THE DEGREE OF \\ Master of SCIENCE \\ IN \\ Computer Science \\ Faculty of Arts And Science \\ LAKEHEAD UNIVERSITY \\ By \\ Xiaodan Tu \\ Thunder Bay, Ontario \\ August 1996
}

(C) Copyright 1996: Xiaodan Tu 
National Library of Canada

Acquisitions and Bibliographic Services

395 Wellington Street Ottawa ON K1A ON4 Canada
Bibliothèque nationale du Canada

Acquisitions et services bibliographiques

395, rue Wellington Ottawa ON KIA ON4

Canada
The author has granted a nonexclusive licence allowing the National Library of Canada to reproduce, loan, distribute or sell copies of this thesis in microform, paper or electronic formats.
L'auteur a accordé une licence non exclusive permettant à la Bibliothèque nationale du Canada de reproduire, prêter, distribuer ou vendre des copies de cette thèse sous la forme de microfiche/film, de reproduction sur papier ou sur format électronique.

L'auteur conserve la propriété du droit d'auteur qui protège cette thèse. $\mathrm{Ni}$ la thèse ni des extraits substantiels de celle-ci ne doivent être imprimés ou autrement reproduits sans son autorisation. 


\section{Table of Contents}

Table of Contents $\quad$ i

List of Tables $\quad$ iii

List of Figures $\quad$ * iv

Chapter 1 INTRODUCTION 1

1.1 Graph Coloring Problem ................... 1

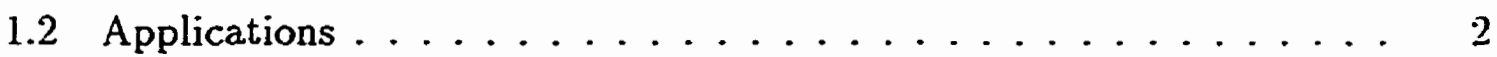

1.3 Overview of the Thesis . . . . . . . . . . . . . . 3

Chapter 2 GRAPH COLORING TECHNIQUES AND PERFECTLY ORDERABLE GRAPHS 4

2.1 NP-Completeness .......................... 4

2.2 Maximal Independent Set Approach . . . . . . . . . . . 5

2.3 Sequential Approach ........................ 7

2.4 Perfectly Orderable Graph . . . . . . . . . . . . . . . . 9

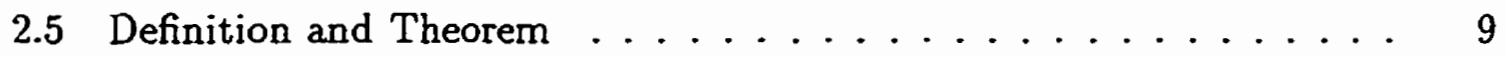

2.6 Recognizing Perfectly Orderable Graphs is NP-Complete . . . . . . . 11

2.7 Orientations and Perfect Orderability .............. 12

2.8 Some Known Subclasses of Perfectly Orderable Graphs . . . . . . . 13 
2.8 .1 Comparability Graphs . . . . . . . . . . . . 13

2.8.2 Triangulated Graphs and Cotriangulated Graphs . . . . 14

$2.8 .3 \quad P_{4}$-Indifference Graphs $\ldots \ldots \ldots \ldots \ldots$

$2.8 .4 \quad P_{4}$-Comparability Graphs $\ldots \ldots . \ldots \ldots \ldots$

$2.8 .5 \quad P_{4}$-Simplicial Graphs $\ldots \ldots \ldots \ldots \ldots \ldots \ldots$

2.8 .6 Raspail Graphs . . . . . . . . . . . . . . 17

2.8.7 Welsh-Powell Perfect Graphs and Matula Perfect Graphs ... 18

2.8.8 Graphs with Dilworth Number at Most Three . . . . . . 19

2.8 .9 The Union of Two Threshold Graphs . . . . . . . . . . 19

2.8 .10 Intersection of Two Threshold Graphs $\ldots \ldots \ldots \ldots . . .22$

2.8 .11 D-Graphs . . . . . . . . . . . . . . 23

2.8 .12 Brittle Graphs. . . . . . . . . . . . . . 23

\section{Chapter 3 INVESTIGATION OF THE PERFECT ORDERABIL-} ITY OF THE UNION OF TWO GRAPHS 25

3.1 Motivation . . . . . . . . . . . . . . . . 25

3.1 .1 The List of Problems . . . . . . . . . . . . . 26

3.1.2 Explanation of the List . . . . . . . . . . . . 26

3.2 The Perfect Orderability of the Union of Two Graphs in Three Cases 30

3.2.1 Some Minimally Non-perfectly Orderable Graphs . . . . . 30

3.2.2 Comparison with Known Classes of Perfectly Orderable Graphs 36

3.2 .3 Conjectures . . . . . . . . . . . . . . . 40

3.3 Proof of the Theorems . . . . . . . . . . . . . 41

3.4 Discussion of the Proof of Theorem $3.3 \ldots \ldots \ldots \ldots$ 


\section{List of Tables}

2.I Six Possible Subclasses of Perfectly Orderable Graphs. . . . . . . 16

3.1 The List of the Union of Two Graphs. . . . . . . . . . . 27 


\section{List of Figures}

2.2 An obstruction. . . . . . . . . . . . . . . 12

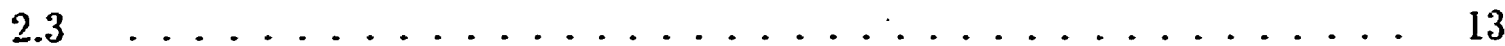

2.4 Transitive orientation. . . . . . . . . . . . 14

2.5 Semi-transitive orientation. . . . . . . . . . . . . . . 14

2.6 Simplicial orientation. . . . . . . . . . . . . . 15

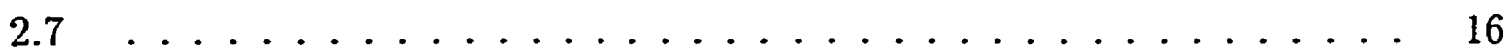

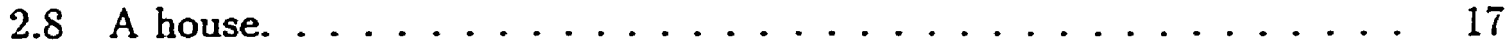

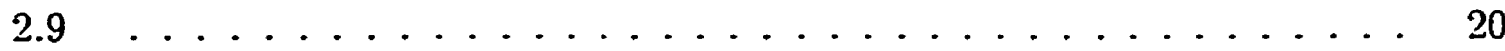

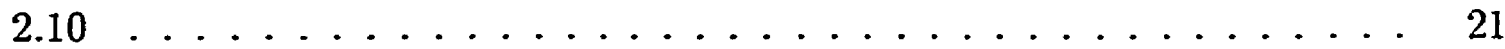

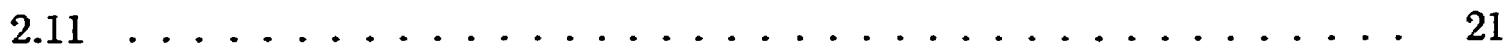

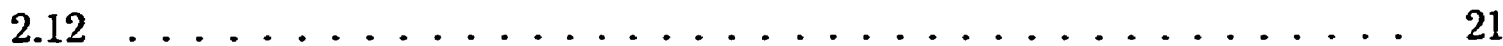

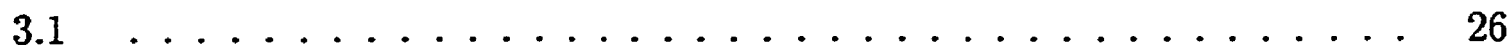

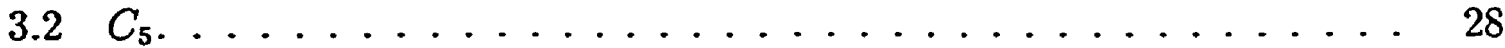

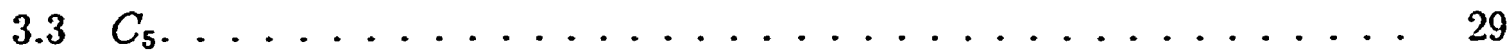

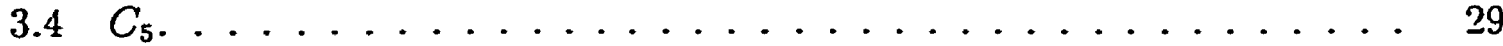

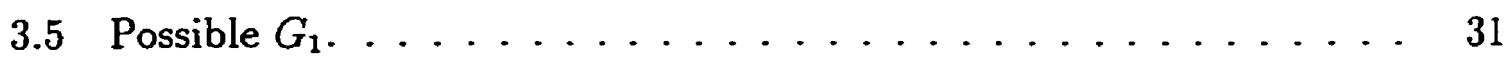

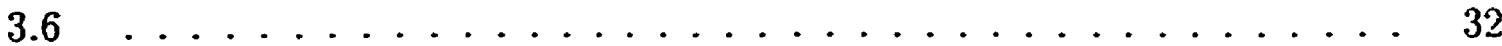

3.7 An anti-hole. $\ldots \ldots \ldots \ldots \ldots \ldots \ldots \ldots \ldots \ldots \ldots \ldots \ldots \ldots \ldots$ 
3.8 Example 1. . . . . . . . . . . . . . . . . 33

3.9 Example $2 . \ldots \ldots \ldots \ldots \ldots \ldots \ldots \ldots \ldots \ldots$

3.10 Example $3 . \ldots \ldots \ldots \ldots \ldots \ldots \ldots \ldots \ldots \ldots \ldots \ldots \ldots \ldots$

3.11 Example 1. . . . . . . . . . . . . . . 36

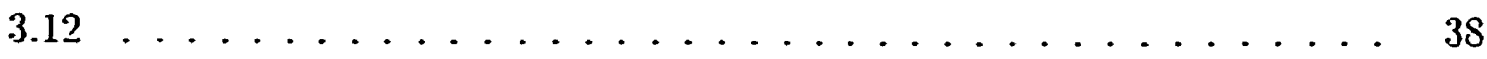

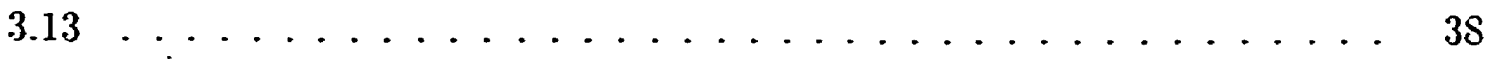

3.14 A non-D-graph. . . . . . . . . . . . . . . . 39

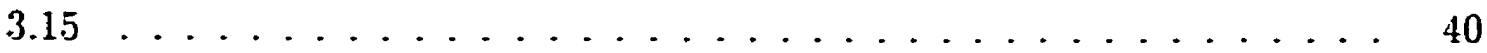

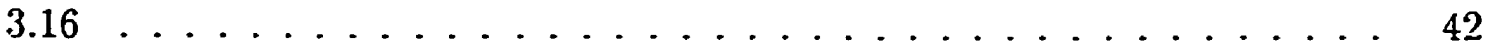

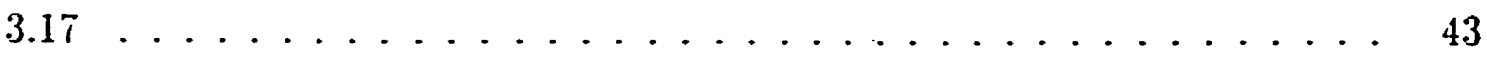

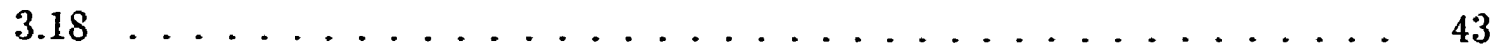

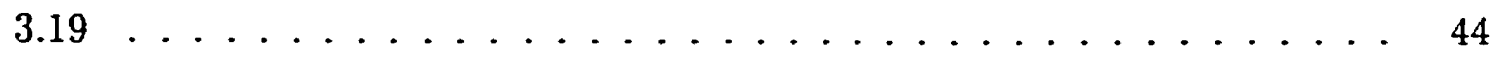

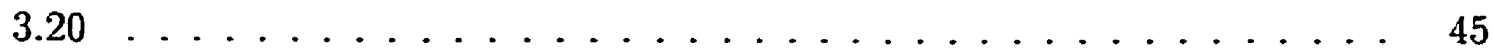

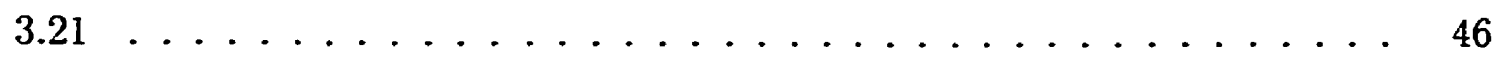

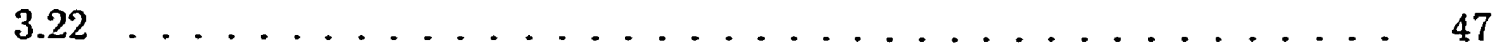

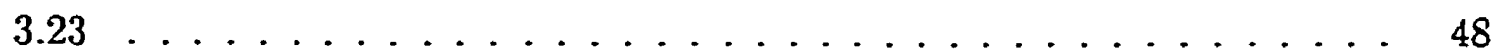

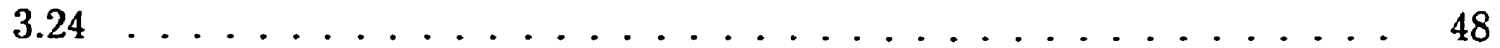

3.25 Two possible $P_{4} \ldots \ldots \ldots \ldots \ldots \ldots \ldots \ldots \ldots$

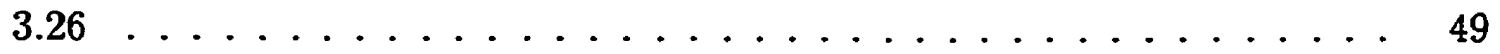

3.27 A counter-example to the rule. . . . . . . . . . . 51

3.28 A counter-example to the question. . . . . . . . . . 52 


\section{ACKNOWLEDGEMENT}

I would like to express my sincere thanks to my thesis supervisor, Dr. C.T. Hoàng,

for his patience, constant encouragement, and guidance in this study. I will remain indebted to him for introducing me to scientific research.

I thank the two anonymous referees for pointing out several mistakes in the first version of this thesis. I would also like to thank others who have helped me in many different ways. The Department of Computer Science gave me academic instructions and provided convenient facilities. My classmates and friends, Jian Wang and Jun Wang, offered me help and advice. The Office of Graduate Studies and Research and my supervisor, Dr. Hoàng, provided financial assistance during the period of my study. My parents and sister gave me special encouragement and care all the time. Without these help, I would find it difficult to undertake and complete this study. To all of them I express my gratitude. 


\begin{abstract}
A graph $G$ is perfectly orderable if it admits an order < on its vertices such that the sequential coloring algorithm delivers an optimum coloring on each induced subgraph $(H,<)$ of $(G,<)$. A graph is a threshold graph if it contains no $P_{4}, 2 \digamma_{2}$. or $C_{4}$ as induced subgraph. A theorem of Chvátal, Hoàng, Mahadev and de Werra states that a graph is perfectly orderable if it can be written as the union of two threshold graphs. In this thesis, we investigate possible generalizations of the above theorem. We conjecture that if $G$ is the union of two graphs $G_{1}$ and $G_{2}$ then $G$ is perfectly orderable whenever (i) $G_{1}$ and $G_{2}$ are both $P_{4}$-free and $2 K_{2}$-free, or (ii) $G_{1}$ is $P_{4}$-free, $2 K_{2}$-free and $G_{2}$ is $P_{4}$-free, $C_{4}$-free. We show that the complement of the chordless cycle with at least five vertices cannot be a counter-example to our conjecture and we prove, jointly with Hoàng, a special case of (i): if $G_{1}$ and $G_{2}$ are two edgedisjoint graphs that are $P_{4}$-free and $2 K_{2}$-free then the union of $G_{1}$ and $G_{2}$ is perfectly orderable.
\end{abstract}




\section{Chapter 1}

\section{INTRODUCTION}

In this chapter, a brief introduction to the graph coloring problem and its application is given, followed by an overview of the thesis.

\subsection{Graph Coloring Problem}

Graph coloring problem is to color the vertices of a graph using a minimum number of colors, subject to the restriction that no two adjacent vertices get the same color. Ever since it was originally formulated in the last century, finding an efficient way to optimally color a graph has attracted the interests of mathematicians. Although many significant results have been derived in this field, it is still left to be one of the most intractable problems in discrete mathematics.

We now give the definition of the graph coloring problem in the term of graph theory:

Definition 1.1 A graph $G$ is said to be r-colorable if its vertices can be colored with $r$ colors in such a way so that no two adjacent vertices are of the same color. The smallest number $r$ for which the graph is $r$-colorable is called the chromatic number $\chi(G)$ of the graph and finding this number is referred to as the coloring problem. 
Here, $G$ is assumed to be a simple graph, that is, an undirected graph with neither loops nor multiple edges.

\section{$1.2 \quad$ Applications}

The earliest application of graph coloring can be traced back to the last century when it was originally formulated. The cartographers asked for a minimum number of colors to color a political map in such a way that no two neighbouring countries get the same color. In this example, different countries can be regarded as the set of vertices $V$ in $G$, and the two vertices of $V$ are adjacent if and only if the two countries they represent are neighbours. They predicted that four colors always suffice. This is known as the Four Color Problem, which was solved by Appel and Haken ([1], [2]) in 1977 .

A contemporary application of the coloring problem is the scheduling and loading problem, as illustrated in the following example.

A computer program stores the values of its variables in memory. For arithmetic computations, the values must be entered in "registers". Registers are expensive, so we want to use them efficiently. If two variables are not used at the same time, we can allocate them to the same register. For each variable, we compute the first and last time it is used. A variable is active during the interval between these times. We define a graph with the variables as vertices, in which two vertices are adjacent if they are active at a common time. The number of registers needed is the optimum coloring of the corresponding graph. Such a graph is called an interval graph:

Definition 1.2 Given any family of intervals, we can define a graph whose vertices are the intervals, with vertices adjacent when the intervals intersect. A graph formed in this way is an interval graph, and the family of intervals is an interval representation of the graph. 
Interval graphs belong to the class of triangulated graphs, the optimum coloring of which can be obtained in polynomial time ([9]). We will discuss triangulated graphs in more detail later.

\subsection{Overview of the Thesis}

This thesis is concerned with the perfect orderability of the union of two graphs. The concept of perfectly orderable graph was developed with the motivation to solve the graph coloring problem. After a brief introduction to the graph coloring problem and its application, in chapter 2, we shall give a brief survey of two graph coloring techniques: maximal independent set and sequential approaches. We shall introduce "perfectly ordered graphs" which are the ordered graphs for which the sequential algorithm delivers an optimum coloring. Chvátal, Hoàng, Mahadev and de Werra proved that a graph is perfectly orderable if it is the union of two threshold graphs. In chapter 3 , we shall investigate possible generalizations of this theorem. In particular, we conjecture that if $G$ is the union of two graphs $G_{1}$ and $G_{2}$ then $G$ is perfectly orderable whenever (i) $G_{1}$ and $G_{2}$ are both $P_{4}$-free and $2 K_{2}$-free, or (ii) $G_{1}$ is $P_{4}$-free, $2 K_{2}$-free and $G_{2}$ is $P_{4}$-free, $C_{4}$-free. We show that the complement of the chordless cycle with at least five vertices cannot be a counter-example to our conjecture and we prove, jointly with Hoàng, a special case of (i): if $G_{1}$ and $G_{2}$ are two edgedisjoint graphs that are $P_{4}$-free and $2 K_{2}$-free then the union of $G_{1}$ and $G_{2}$ is perfectly orderable. 


\section{Chapter 2}

\section{GRAPH COLORING TECHNIQUES AND PERFECTLY ORDERABLE GRAPHS}

In this chapter, a further discussion of the graph coloring is given. Despite the NP. complete property of this problem, we introduce two basic approaches to color a graph: maximal independent set and sequential, followed by a more detailed addressing on the concept and study of perfectly orderable graphs.

\section{$2.1 \quad$ NP-Completeness}

The coloring problem was proved to belong to the class of NP-complete problems ([7]), and worse than that, a polynomial approximation algorithm with a constant error ratio cannot exist unless $P=N P([16])$.

As a result of its complexity, although the number of papers on the coloring problem exceeds that on any other graph problem, no formula has been found for the chromatic number of an arbitrary graph and we must thus be satisned with bound estimates. Here, we are going to introduce only two simple bounds: 


$$
\text { i)lower bound : } \quad \omega(G) \leq \chi(G)
$$

$\omega(G)$ is the number of vertices in the largest clique of $G$. Since all vertices of any clique of $G$ must have different colors, this lower bound is obvious.

$$
\text { ii)upper bound : } \quad \chi(G) \leq \Delta(G)+1
$$

$\Delta(G)$ denotes the maximum degree of a vertex of $G$. This inequality follows from the observation that if $\Delta(G)+1$ colors are available, then at each vertex $v$ of the graph $G$ at least one of the colors can be used, since at most $\Delta(G)$ colors are used to color the neighbours of $v$. Brooks ([18]) proved further that there are only two classes of graphs for which the upper-bound holds with equality: odd cycles and complete graphs.

Despite the great difficulty, many efforts have been given to tackle the coloring problem. There are basicly two approaches: independent set and sequential.

\subsection{Maximal Independent Set Approach}

A k-coloring of $G$ is a partition of the set of vertices of $G$ into $k$ independent sets $V_{1}, V_{2}, \ldots, V_{k}$ such that $V_{i} \cap V_{j}=\emptyset$ for $i \neq j, i, j=1,2, \ldots, k$, and $\bigcup_{i=1}^{k} V_{i}=V$. Such a partition is called a $k$-coloring partition of $V$. Thus, the coloring problem is equivalent to finding a minimum $k$ in a $k$-coloring partition of $G$.

Let $V_{1}, V_{2}, \ldots, V_{k}$ be a $k$-coloring partition of a $k$-colorable graph $G$. Then we can construct a $l$-coloring partition $V_{1}^{\prime}, V_{2}^{\prime}, \ldots, V_{l}^{\prime}$ of $G$ such that $V_{1}^{\prime}$ is a maximal (in the sense of set-inclusion) independent set of $G$, and $l \leq k$ in the following way: First set $V_{1}^{\prime}=V_{1}$, and for each vertex $x$ in $V_{2} \cup V_{3} \ldots \cup V_{k}$. we put $x$ in $V_{1}^{\prime}$ if and only if $x$ has 
no neighbour in $V_{1}^{\prime}$. Then we set $V_{i}^{\prime}=V_{i}-\left\{x \mid x \in V_{1}^{\prime}\right\}$ for $2 \leq i \leq k$. It follows that there exists a maximal independent subset $U$ of the vertices of $G$ such that

$$
\chi(G)=\chi\left(G_{V-U}\right)+1
$$

( $G_{V-U}$ is the subgraph of $G$ induced by $V-U$. .)

There is a finite number of maximal independent sets $W$ in $G$. minimizing over all such subsets, we obtain

$$
\chi(G)=\min _{W \subseteq U} \chi\left(G_{V-W}\right)+1
$$

Equation 2.4 is the basis for the following algorithm (Maximal Independent Set approach, or MIS) for computing the chromatic number: ${ }^{\circ}$

Procedure $\operatorname{MIS}(G, k)$;

Input: a non-empty graph $G$.

output: chromatic number $k$ of $G$.

begin \{procedure\}

$$
\text { if } G=\emptyset \text { then } k:=0
$$

else

begin

$$
k:=|V(G)|
$$

for each maximal independent set $S$ of $G$ do

begin

$$
\begin{aligned}
& \operatorname{MIS}(G-S, l) \\
& \text { if }(l+1)<k \text { then } k:=l+1 ;
\end{aligned}
$$

end;

end;

end; \{procedure 
It is easy to see that procedure MIS can be modified to produce a $k$-coloring of a $k$-chromatic graph. The complexity of this method is $O\left(m n 2.445^{n}\right)$ (see [19], as usual $n$ and $m$ denote the number of vertices and edges respectively).

\subsection{Sequential Approach}

Coloring function is often used when discussing the sequential approach for coloring a graph.

Definition 2.1 A function $f$ determines a $k$-coloring of $G$. if

$$
f: V \rightarrow\{1,2, \ldots, k\}
$$

with $f(i) \neq f(j)$ for all $(i, j) \in E$. A function that defines a $k$-coloring is called the $k$-coloring function.

A sequential approach can be stated as the following greedy algorithm:

Algorithm (Greedy coloring) The greedy coloring with respect to a vertex ordering $v_{1}, \ldots, v_{n}$ of $V(G)$ is obtained by coloring vertices in the order $v_{1}, \ldots, v_{n}$, assigning to $v_{i}$ the smallest-indexed color not already used on its lower-indexed neighbours.

From the above sequential approach, it is easy to determine an upper bound $u_{s}\left(G ; v_{1}, v_{2}, \ldots, v_{n}\right)$ for the number of colors $\chi_{s}(G)$ used by the sequential algorithm applied to $G$ and the ordering of its vertices $v_{1}, v_{2}, \ldots, v_{n}$. Every vertex $v_{i}$ can be colored by color $i$, therefore $f\left(v_{i}\right) \leq i$. On the other hand, at least one of the first $\operatorname{deg}\left(v_{i}\right)+1$ colors can be assigned to $v_{i}$. Hence,

$$
f\left(v_{i}\right) \leq \min \left\{i, \operatorname{deg}\left(v_{i}\right)+1\right\}
$$


for every $i=1,2, \ldots, n$, and thus

$$
\chi_{s}(G) \leq u_{s}\left(G ; v_{1}, v_{2}, \ldots, v_{n}\right)=\max _{i \leq n} \min \left\{i, \operatorname{deg}\left(v_{i}\right)+1\right\}
$$

This inequality is obtained without any assumption about the ordering of vertices of $G$.

It is obvious that in a sequential approach, the key point is to order the vertices of $G$, because it exclusively decides the behaviour of the greedy algorithm.

Welsh and Powell ([6]) gave the first version of sequential method by ordering the vertices according to nonincreasing degree, $\operatorname{deg}\left(v_{1}\right) \geq \operatorname{deg}\left(v_{2}\right) \geq \ldots \geq \operatorname{deg}\left(v_{n}\right)$. Such an ordering is called the largest-first ordering, or LF.

A closer inspection of the algorithm and the proof of the inequality(2.7) reveals that for a given ordering $v_{1}, v_{2}, \ldots, v_{n}$ of the vertices of a graph $G$, instead of $f\left(v_{i}\right) \leq$ $1+\operatorname{deg}\left(v_{i}\right)$ we have in fact $f\left(v_{i}\right) \leq 1+\operatorname{deg}_{i}\left(v_{i}\right)$, where $\operatorname{deg}_{i}\left(v_{i}\right)$ denotes the degree of vertex $v_{i}$ in the subgraph of $G$ induced by $v_{1}, v_{2}, \ldots, v_{i}$. Therefore, the algorithm never requires more than $\max \left\{1+\operatorname{deg}_{i}\left(v_{i}\right): 1 \leq i \leq n\right\}$ colors; hence

$$
\chi_{s}(G) \leq u_{s}^{\prime}\left(G ; v_{1}, v_{2}, \ldots, v_{n}\right)=\mathrm{I}+\max _{1 \leq i \leq n} \operatorname{deg}_{i}\left(v_{i}\right)
$$

The following procedure finds a vertex ordering which minimizes $u_{s}^{\prime}\left(G ; v_{1}, v_{2}, \ldots, v_{n}\right)$ :

1. $v_{n}$ is a minimum degree vertex of $G$.

2. For $i=n-1, n-2, \ldots, 2,1, v_{i}$ is a minimum degree vertex in the subgraph of $G$ induced by $V-\left\{v_{n}, v_{n-1}, \ldots, v_{i+1}\right\}$.

Such an ordering is called smallest-last SL.

Both LF and SL algorithms may improve the upper bound (2.2) substantially. But neither of them can guarantee an optimum coloring of an arbitrary graph $G$. It 
is natural for one to ask such a question: for which kind of ordered graphs does the greedy algorithm produce an optimum coloring? (An ordered graph is a graph with a given total order $<$ on its vertices.) This is what motivated V.Chvátal to propose the concept of perfectly orderable graph.

\subsection{Perfectly Orderable Graph}

In this section, we introduce the concept of perfectly orderable graph, and the well-known theorem developed by Chvátal that reveals the equivalent nature of perfectly orderability and admissive order. Knowing that to recognize perfectly orderable graphs is $N P$-complete, we introduce some subclasses of perfectly orderable graphs. many of which can be recognized in polynomial time.

\subsection{Definition and Theorem}

Definition 2.2 (Chvátal [3]) An obstruction in an ordered graph $(G,<)$ is a set of four vertices $a, b, c, d$ with edges $a b, b c, c d$ (and no other edges) and $a<b, d<$ c. A linear order on the set of vertices of a graph will be called i) admissible if it creates no obstruction and ii) perfect if, for each induced ordered subgraph $H$, the greedy algorithm produces an optimum coloring of $H$. A graph will be called perfectly orderable if it admits a perfect order.

Chvátal also revealed the nature between perfect order and admissible order by proving the following theorem, which becomes the criterion for identifying perfectly orderable graphs:

Theorem 2.1 (Chvátal [3]) A linear order of the set of vertices of a graph is perfect if and only if it is admissible. 
Proof: Since the class of graphs having obstruction-free orderings is hereditary (the inherited ordering for an induced subgraph is obstruction-free), it suffices to show that an obstruction-free ordering $L$ gives a greedy coloring of $G$ that is optimum. If the greedy coloring uses $k$ colors for the ordering $L$, then optimality can be established by showing that $G$ has a $k$-clique (a clique on $k$ vertices). To show that such a clique does exist, we introduce the following lemma:

Lemma 2.1 Suppose that $G$ has a clique $Q$ and a stable set $S$ disjoint from $Q$, and suppose that for each vertex $w \in Q$ there is a vertex $p(w) \in S$ such that $p(w)$ and $w$ are adjacent. If $L$ is an obstruction-free ordering of $G$ such that $p(w)<w$ for all $w \in Q$, then some $p(w) \in S$ is adjacent to all of $Q$.

Proof: By induction on $|Q|$. The lemma holds trivially for $|Q|=1$, so we may assume $|Q|>1$. For each $w \in Q$, the graph $Q-w$ satisfies the hypotheses using the clique $Q-w$ and the stable set $\{p(u): u \in Q-w\}$. By the induction hypothesis there is a vertex $w^{*} \in Q-w$ such that $p\left(w^{*}\right)$ is adjacent to all $Q-w$. We may assume that $p\left(w^{*}\right)$ is not adjacent to $w$ for every $w \in Q$ (for otherwise, $p\left(w^{*}\right)$ is adjacent to all of $Q$ and we are done). This assigns a unique $w^{*}$ to every $w$, since $p\left(w^{*}\right)$ is nonadjacent only to $w$ among $Q$. So setting $\sigma(w)=w^{*}$ defines a bijection $\sigma$ on the vertices of $Q$.

Let $v$ be the least vertex of $Q$ in $L$. Let $b, c \in Q$ be the vertices such that $b^{*}=v$ and $c^{*}=b$. Let $a=p(b)$ and $d=p(v)$. Because $p\left(w^{*}\right)$ is not adjacent to $w$ and $p(w)<w$, we have that $d$ is not adjacent to $b, a$ is not adjacent to $c$, and $d<v$ and $a<b$. Since $a, d$ belong to the stable set $S, a$ is not adjacent to $d$ either (see the Figure 2.1 bellow. An oriented edge of the form $x \rightarrow y$ means $x<y$ and $x y \in E$.)

Because $d=p\left(b^{*}\right)$, the only vertex of $Q$ nonadjacent to $d$ is $b$, which implies that $c$ is adjacent to $d$. Since $d=p(v)<v<c$ in $L$, the edge $d c$ is oriented as $d \rightarrow c$, which implies that $a, b, c$ and $d$ induce an obstruction, a contradiction.

Now we continue to prove the Theorem 2.1. 


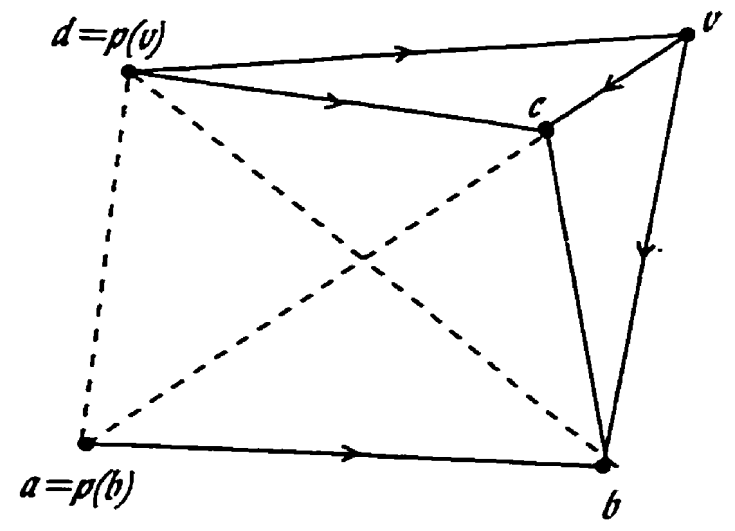

Figure 2.1

Let $f: V(G) \rightarrow\{1,2, \ldots, k\}$ be the coloring generated by the greedy algorithm with this ordering. Let $i$ be the smallest integer such that $G$ has a clique consisting of vertices $w_{i+1}, \ldots, w_{k}$ such that $f\left(w_{j}\right)=j$. Since $f$ uses $k$ on some vertex, such a clique exists. If $i=0$, then $G$ has a $k$-clique. Suppose $i>0$. For each $w_{j}$ there is a vertex $p\left(w_{j}\right)$ such that $p\left(w_{j}\right)<w_{j}$ in $L$ and $f\left(p\left(w_{j}\right)\right)=i$; otherwise the greedy coloring would have used a lower color on $w_{j}$. Since the vertices in $S=\left\{p\left(w_{i+1}\right), \ldots, p\left(w_{k}\right)\right\}$ all have color $i, S$ is a stable set. Hence, the conditions of the Lemma 2.1 are satisfied, and there is a vertex of $S$ that can be added to the clique and called $w_{i}$, which contradicts the minimality of $i$.

Perfectly orderable graphs generalize many well-known classes of graphs, such as comparability graphs, triangulated graphs and their complements. We shall discuss this fact later.

\subsection{Recognizing Perfectly Orderable Graphs is NP-Complete}

When Chvátal introduced the notion of perfectly orderable graphs, he also posed the question: how difficult is it to recognize perfectly orderable graphs? Middendorf 
and Pfeiffer answered this question by proving the following theorem:

Theorem 2.2 (Middendorf, Pfeiffer [17]) To decide whether a graph admits a perfect order is NP-complete.

They proved the theorem by giving a reduction of 3SAT to the problem of deciding whether a graph admits a perfect order.

\subsection{Orientations and Perfect Orderability}

When studying perfectly orderable graphs, sometimes it is convenient to work with orientations instead of orders.

Definition 2.3 An orientation ${ }^{(i}$ of a graph $G$ is a directed graph obtained from $G$ by assigning a direction to each edge of $G$.

To an ordered graph $(G,<)$, there corresponds an orientation $D(G,<)$ of $G$ such that $\overrightarrow{a b} \in D(G,<)$ if and only if $a b \in E(G)$ and $a<b$. So a graph is perfectly orderable if and only if it admits an acyclic orientation that does not contain an induced subgraph isomorphic to the Figure 2.2:

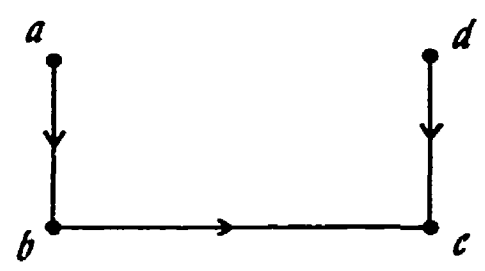

Figure 2.2: An obstruction.

Equivalently: a graph is perfectly orderable if and only if it admits an acyclic orientation in which each induced path of length three is one of the three types in Figure 2.3: 

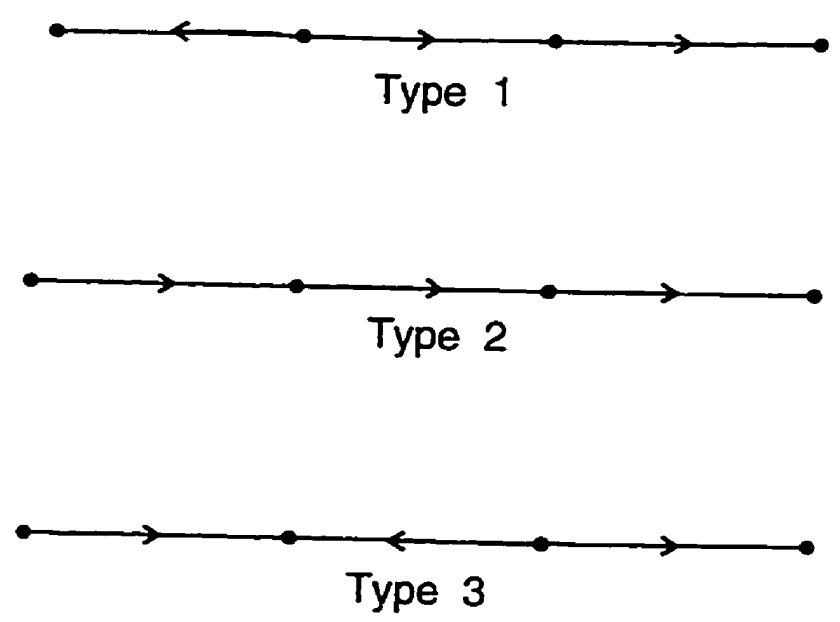

Figure 2.3

Although the class of perfectly orderable graphs has very nice properties in the sense of optimization, there is no known polynomial-time algorithm to recognize it. However, many subclasses of perfectly orderable graphs with special characteristics have been studied, many of which can be recognized in polynomial time. Next, we are going to introduce some of the known classes of perfectly orderable graphs.

\subsection{Some Known Subclasses of Perfectly Orderable Graphs}

\subsubsection{Comparability Graphs}

Definition 2.4 A simple graph $G$ is a comparability graph if it has a transitive orientation, which is an acyclic orientation such that if $x y,-y z \in E(G), x \rightarrow y$ and $y \rightarrow z$, then $x z \in E(G)$ and $x \rightarrow z$.

From Definition 2.4, a transitive orientation implies that each induced $P_{3}$ in a comparability graph is of the types in Figure 2.4, and it is clear there is no obstruction. So comparability graphs are perfectly orderable. 


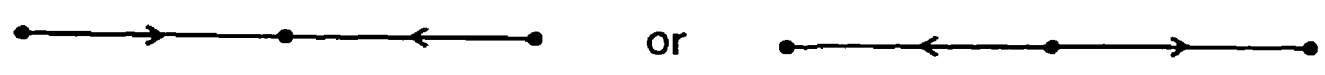

Figure 2.4: Transitive orientation.

The following theorem of Ghouila-Houri $([8])$ is the key to a polynomial-time algorithm to recognize comparability graphs.

Theorem 2.3 A graph is a comparability graph if and only if it admits an orientation that contains no induced subgraph isomorphic to the graph in Figure 2.5 (a semitransitive orientation).

Note that in the above theorem, the orientation may contain directed cycles.

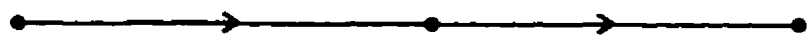

Figure 2.5: Semi-transitive orientation.

\subsubsection{Triangulated Graphs and Cotriangulated Graphs}

Definition 2.5 A triangulated graph $G$ is a graph such that every cycle of length $\geq 4$ in $G$ has a chord, that is, an edge joining two non-consecutive vertices of the cycle. It is also called chordal, rigid-circuit, monotone transitive, and perfect elimination graph.

A triangulated graph $G$ has the property that every induced subgraph contains a vertex whose neighbourhood induces a clique (a simplicial vertex). It follows that it admits an order $<$, such that $v_{i}$ is simplicial in the subgraph induced by $H=$ 
$\left\{x \mid x<v_{i}\right\} \cup\left\{v_{i}\right\}$. Such an order is called a simplicial order. In a simplicial order, each $P_{3}$ is of the types shown in Figure 2.6. So it is obvious that a simplicial order is a perfect order. Testing whether a graph is triangulated is polynomially equivalent to testing whether a graph contains a simplicial vertex which can be obviously solved in polynomial time.

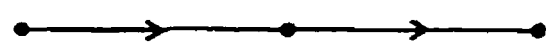

or

Figure 2.6: Simplicial orientation.

Definition 2.6 A cotriangulated graph is the complement of a triangulated graph.

In a triangulated graph $G$, the simplicial order implies that each $P_{4}$ is of the Type 1 or 2 in Figure 2.3. In the complement $\bar{G}$ of $G$, define an order $<_{\bar{G}}$ such that $x<{ }_{\bar{G}} y$ if and only if $y<_{G} x$. We shall show that the order $<_{\bar{G}}$ contains no obstruction.

By $a b c d$, we denote the $P_{4}$ with vertices $a, b, c, d$, edges $a b, b c, c d$ (and no other edges). If $a b c d$ is a $P_{4}$ of $G$, then its complement in $\bar{G}$ is bdac. Suppose $a b c d$ is a $P_{4}$ of Type $I$ in $G$ (see Figure 2.7 (a)). Without loss of generality, we may assume that $b<_{G} a, b<_{G} c, c<_{G} d$. The last two relations imply that $b<_{G} d$, and therefore $d<_{\bar{G}} b$. Thus $b d a c$ is not an obstruction in $\bar{G}$. A similar argument shows that the complement of a $P_{4}$ of Type 2 in $G$ cannot be an obstruction (Figure 2.7 (b)).

By restricting the orientation on $P_{4}$ to each of the three types in Figure 2.3, Hoàng and Reed ([15]) introduced the following six subclasses of perfectly orderable graphs, four of which can be recognized in polynomial time. 

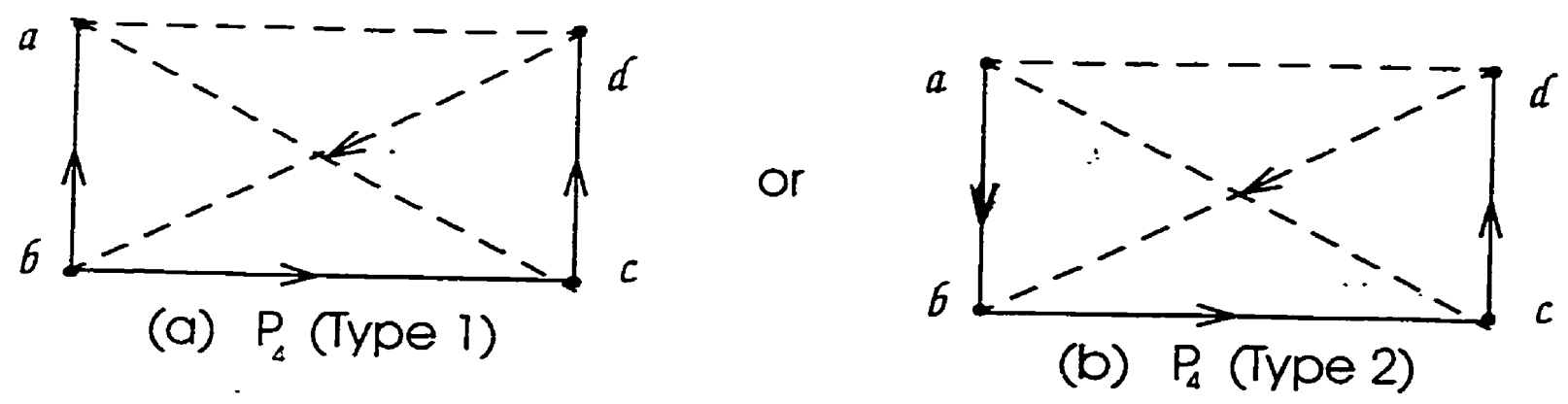

Figure 2.7

\begin{tabular}{||c|l|l|l|l|r||}
\hline & \multicolumn{3}{|c|}{$P_{4}$ allowed in $G$} & \multirow{2}{*}{ Name } & $\begin{array}{r}\text { Recognition } \\
\text { Complexity }\end{array}$ \\
\cline { 2 - 6 } & Type 1 & Type 2 & Type 3 & & Polynomial \\
\hline 1 & $\sqrt{ }$ & $\sqrt{ }$ & & $P_{4}$-simplicial & N \\
\hline 2 & $\sqrt{ }$ & & $\sqrt{ }$ & Generalized CR & NP-Complete \\
\hline 3 & & $\sqrt{ }$ & $\sqrt{ }$ & One-in-one-out & Unknown \\
\hline 4 & $\sqrt{ }$ & & & Raspail & Palynomial \\
\hline 5 & & $\sqrt{ }$ & & $P_{4}$-indifference & Polynomial \\
\hline 6 & & & $\sqrt{ }$ & $P_{4}$-comparability & Polynomial \\
\hline
\end{tabular}

Table 2.1: Six Possible Subclasses of Perfectly Orderable Graplıs.

\subsection{3 $\quad P_{4}$-Indifference Graphs}

Definition 2.7 $A$ graph $G$ is $P_{4}$-indifference if there is an acyclic orientation of $G$ in which every $P_{4}$ is of Type 2.

\subsection{4 $P_{4}$-Comparability Graphs}

Definition 2.8 $A$ graph $G$ is $P_{4}$-comparability if it admits an acyclic orientation in which each $P_{4}$ is of Type 3.

In a transitive orientation, every $P_{4}$ is of Type 3 . Thus the class of $P_{4}$-comparability graphs contains all comparability graphs. 


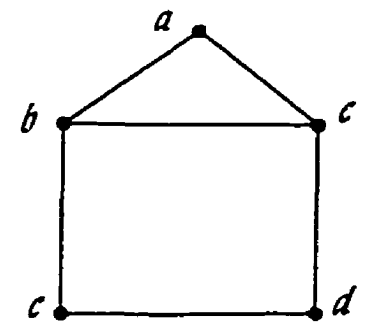

Figure 2.8: A house.

\subsection{5 $\quad P_{4}$-Simplicial Graphs}

Definition 2.9 A graph $G$ is $P_{4}$-simplicial if there is an acyclic orientation of $G$ in which every $P_{4}$ of $G$ is of Type 1 or 2.

In a simplicial order, every $P_{4}$ is of Type 1 or 2 . Thus the class of $P_{4}$-simplicial graphs contains all triangulated graphs.

\subsubsection{Raspail Graphs}

Definition 2.10 A graph is Raspail if it admits an acyclic orientation in which every $P_{4}$ is of Type 1.

Hertz and de Werra ([11]) gave a characterization of Raspail graphs by forbidding induced subgraphs, among which is the house, as illustrated in Figure 2.8.

It is easy to see that in a house $a b c d e$, the two $P_{4} \mathrm{~s} a b c d$ and $c d e a$ cannot be both of Type 1. We shall refer to this property of the house later in the thesis.

Hoàng and Reed ([15]) also developed polynomial-time algorithms to recognize the above four classes of perfectly orderable graphs.

Hoàng ([13]) also showed that recognizing "generalized CR" graphs is NP-complete.

It is not known whether "one-in-one-out" graphs can be recognized in polynomial time. 


\subsubsection{Welsh-Powell Perfect Graphs and Matula Perfect Graphs}

We consider graphs $G$ with linear orders < on the vertices. Welsh and Powell choose $<$ in such a way that,

$$
d_{G}(x) \geq d_{G}(y) \text { whenever } x<y
$$

with $d_{G}(x)$ standing for the degree of $x$ in $G$;

while Matula chooses < in such a way that,

$$
d_{H}(x) \geq d_{H}(y) \text { whenever } x<y
$$

and $H$ is the subgraph of $G$ induced by all $z$ with $z \leq y$.

Definition 2.11 $G$ is called Welsh-Powell perfect if the linear order < satisfying (2.9) is perfect (the greedy algorithm produces an optimum coloring for each induced subgraph of $G$ ), and Matula perfect if the linear order < satisfying (2.10) is perfect.

Chvátal, Hoàng, Mahadev and de Werra ([5]) proved theorems to characterize Welsh-Powell perfect and Matula perfect graphs by forbidding certain induced subgraphs. Again a house is such a forbidden subgraph for both Welsh-Powell and Matula perfect graphs. They also showed that these two classes of perfectly orderable graphs can be recognized in polynomial time, and presented an algorithm to prove the following theorem:

Theorem 2.4 Given any graph $G$ that is Welsh-powell perfect or Matula perfect, one can find in time $O(m+n)$ a minimum coloring and a largest clique in $G$. Given any graph $G$ whose complement is Welsh-Powell perfect or Matula perfect, one can find in time $O(m+n)$ a minimum clique cover and a largest stable set in $G$. 


\subsubsection{Graphs with Dilworth Number at Most Three}

Definition 2.12 Let $N(x)$ stand for the set of all the neighbours of a vertex $x$ in $G$; we say that a vertex $y$ dominates $x$ if $N(x) \subseteq N(y) \bigcup\{y\} . x$ and $y$ are comparable if $x$ dominates $y$ or $y$ dominates $x$, and incomparable if neither of them dominates the other. The Dilworth number of $G$ is the largest number of pairwise incomparable vertices in $G$.

It is obvious that the class of graphs with Dilworth number at most three is recognizable in polynomial time. In [5], it is proved that these graphs are perfectly orderable.

\subsubsection{The Union of Two Threshold Graphs}

Threshold graphs were introduced to study the stable set polytope of a graph.

Definition 2.13 Let $G=(V, E)$ be a graph on $n$ vertices. Then $G$ is a threshold graph if there exists a linear inequality

$$
\sum_{i=1}^{n} a_{i} x_{i} \leq a
$$

with $a, a_{i} \in \Re$ and $n=|V|$ such that the following holds: $S \subset V$ is a stable set of $G$ if and only if (2.11) is satisfied by the characteristic vector $x_{s}=\left(x_{1}, x_{2}, \ldots, x_{n}\right)$ of $S$ where for all $i$

$$
x_{i}= \begin{cases}1 & \text { if } i \in S \\ 0 & \text { if } i \notin S\end{cases}
$$

Theorem 2.5 (Chvátal, Hammer [4]) A necessary and sufficient condition for $G$ to be threshold is that $G$ does not contain $2 K_{2}, P_{4}$ or $C_{4}$ as an induced subgraph (Figure 2.9.) 


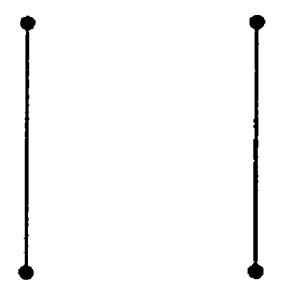

$2 K_{2}$

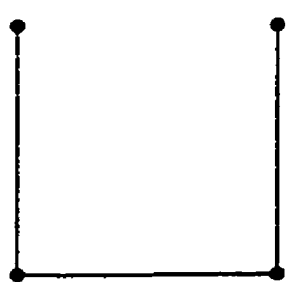

$P_{4}$

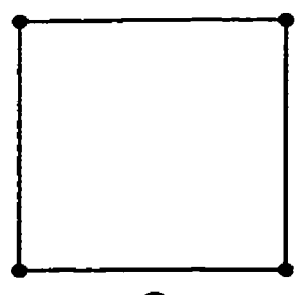

C،

Figure 2.9

Chvátal, Hoàng, Mahadev and De Werra proved the following theorem:

Theorem 2.6 If $G$ is the union of two threshold graphs then $G$ is perfectly orderable.

We are going to give a new proof of the Theorem 2.6. But before that, let us first give some definitions about $P_{4}$ : Let $a b c d$ denote the $P_{4}$ with vertices $a, b, c, d$ and edges $a b, b c, c d$, the edges $a b$ and $c d$ are called wings of $P_{4}$.

Proof: Let $G$ be the union of two threshold graphs $G_{1}$ and $G_{2}$. Consider any $P_{4}$ $v_{0} v_{1} v_{2} v_{3}$ in $G$ (if it exists). It is clear that the two wings $v_{0} v_{1}$ and $v_{2} v_{3}$ cannot be in the same $G_{i}(i=1,2)$, otherwise there will be either a $2 K_{2}$ or a $P_{4}$ in $G_{i}$.

$\forall P_{4} \in G$, we now impose a partial orientation on the edges of $G$ :

1. direct $v_{1} \rightarrow v_{0}$ if and only if $v_{0} v_{1} \in G_{i}$ and $v_{1} v_{2} \in G_{i}$,

2. direct $v_{2} \rightarrow v_{3}$ if and only if $v_{1} v_{2} \in G_{i}$ and $v_{2} v_{3} \in G_{i}$.

It is obvious that such a partial orientation does not create an obstruction in $G$.

Claim 2.1 Such an orientation is unique on each edge.

Proof: Suppose there is an edge $c d$ that receives two directions by the above orientation.

Let us assume $c d \in G_{1}$. We must have a $P_{4}$ abcd with $a b \in G_{2}, b c \in G_{1}$ and another $P_{4}$ cdef with $d e \in G_{1}$, ef $\in G_{2}$. Since $b d \notin G_{1}$ and $c e \notin G_{1},\{b, c, d, e\}$ form either a $P_{4}$ or $C_{4}$ in $G_{1}$, a contradiction (see Figure 2.10.) $\square$ 
(In all of the following figures in this thesis, we use solid lines to represent $G_{1}$ and dashed lines to represent $G_{2}$.)

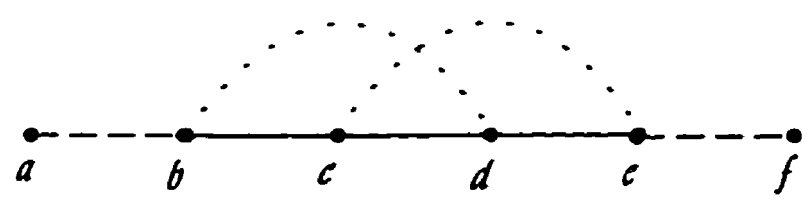

Figure 2.10

Claim 2.2 There are no two directed edges $\overrightarrow{a b}, \overrightarrow{b c}$ (Figure 2.11) such that $\overline{a b} \in G_{1}$ (respectively $G_{2}$ ) and $\overrightarrow{b c} \in G_{2}$ (respectively $G_{1}$ ).

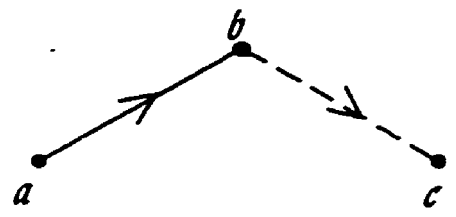

Figure 2.11

Proof: Suppose there are directed edges $\overrightarrow{a b}, \overrightarrow{b c}$ with $\overrightarrow{a b} \in G_{1}$ and $\overrightarrow{b c} \in G_{2}$.

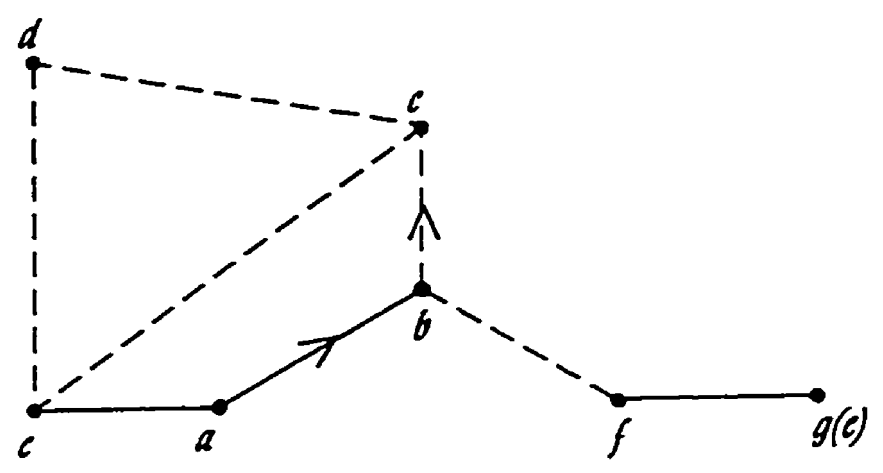

Figure 2.12

Since $a \rightarrow b$ in $G_{1}$, there must be a $P_{4}$ deab with $d e \in G_{2}$, and $e a, a b \in G_{1}$ ( $c$ is not identical to either $d$ or $e$ ); similarly, since $b \rightarrow c$ in $G_{2}$, there must be a $P_{4} g f b c$ 
with $g f \in G_{1}$ and $f b, b c \in G_{2}$ ( $g$ may be identical to $e$, but $f$ cannot be identical to either $d$ or $e$ ). Since $G_{2}$ is a threshold graph, to have both edges $e d$ and $b c$ belonging to $G_{2}$, there must be $c e \in G_{2}$ and $c d \in G_{2}$. Now consider the subgraph consisting of the three edges $d c, c b$ and $b f$ in $G_{2}$. We have $d b \notin E(G)$ and $c f \notin E(G)$. There will be either an induced $P_{4}$ (if $d f \notin E\left(G_{2}\right)$ ) or $C_{4}$ (if $d f \in E\left(G_{2}\right)$ ) in $G_{2}$, a contradiction (see Figure 2.12.)

Claim 2.3 The partial orientation creates no directed cycle in $G$.

Proof: We note that for any directed edge $\overrightarrow{a b} \in G_{i}, a$ strictly dominates $b$ in $G_{i}$ ( $a$ dominates $b$, but $b$ does not dominate $a$ ). Therefore, there will be no directed cycle in the same $G_{i}$. Furthermore, Claim 2.2 guarantees that there is no directed cycle consisting of edges in both $G_{1}$ and $G_{2}$.

Now wa can easily extend this acyclic partial orientation to a linear order $<$ on $G$ by the following procedure:

1. $i \leftarrow 1, H \leftarrow G$;

2. Find a vertex $v$ with indegree (the number of directed edges pointing to $v$ ) 0 in $H$, assign to it the number $i$. If no such vertex $v$ exists, order the remaining vertices in $H$ randomly and then STOP.

3. $H \leftarrow H-v, i \leftarrow i+1$, if $H$ is not empty, goto step 2 .

Such an order $<$ is obstruction-free and thus is perfect. Therefore, $G$ is perfectly orderable.

\subsubsection{Intersection of Two Threshold Graphs}

Definition 2.14 A graph $G$ is the intersection of two threshold graphs if there are two threshold graphs $G_{1}$ and $G_{2}$ such that $e \in E(G) \Longleftrightarrow e \in E\left(G_{1}^{\prime}\right)$ and $e \in E\left(G_{2}\right)$. 
Hammer and Mahadev proved [10] that intersections of two threshold graphs are perfectly orderable.

\subsubsection{D-Graphs}

Definition $2.15 A$ vertex $x$ is a d-vertex if for any edge $y z$ with $\{y, z\} \cap N(x)=\emptyset$. $y$ and $z$ are comparable. A graph $G$ is a D-graph if each of its induced subgraphs contains a d-vertex.

Hoàng ([12]) showed that the class of $D$-graphs contains all graphs with the Dilworth number at most three and all cotriangulated graphs. He also proved that every $D$-graph is perfectly orderable, and used this result to prove the the following theorem:

Theorem 2.7 Let $G_{1}$ be a threshold graph and let $G_{2}$ be a graph containing no induced $P_{4}$ and no induced $C_{4}$. Then the union of $G_{1}$ and $G_{2}$ is perfectly orderable.

A polynomial algorithm with the complexity of $O(n m)$ has been developed to recognize $D$-graphs ([12]).

\subsubsection{Brittle Graphs}

Definition 2.16 Let abcd be a $P_{4}$ of a graph $G$. The vertices a, $d$ are called endpoints of the $P_{4}$ and the vertices $b, c$ are called midpoints of the $P_{4} . G$ is called brittle if each induced subgraph $H$ of $G$ contains a vertex which is not an endpoint or a midpoint of - $a P_{4}$ in $H$.

Chvátal introduced brittle graphs and pointed out that they are perfectly orderable. For more information on brittle graphs, see ([14]). The class of brittle graphs 
contains all $P_{4}$-simplicial graphs, all $P_{4}$-indifference graphs, all triangulated graphs and their complements, all Raspail graphs, all Welsh-Powell perfect graphs, all Matula perfect graphs and all graphs with Dilworth number at most three. 


\section{Chapter 3}

\section{INVESTIGATION OF THE PERFECT ORDERABILITY OF THE UNION OF TWO GRAPHS.}

With the motivation to generalize Theorem 2.6 (that the union of two threshold graphs is perfectly orderable), we study the perfect orderability of the unions of the two graphs, in which some or all of the three induced subgraphs $P_{4}, C_{4}$ and $2 K_{2}$ are forbidden. For three of the all 28 possible cases of the unions, we pose the conjecture that they form a new subclass of perfectly orderable graphs. Two theorems are proved to support our conjecture.

\subsection{Motivation}

In the last chapter, we introduced a subclass of perfectly orderable class, the union of the two threshold graphs, and also mentioned that by allowing a $2 K_{2}$ in one of the graphs, Hoàng used the property of $D$-graph to prove the Theorem 2.7, which generalizes the Theorem 2.6 .

We know that a threshold graph is a graph that contains no $P_{4}, 2 K_{2}$ or $C_{4}$ as an induced subgraph. Given two graphs $G_{1}$ and $G_{2}$, Theorem 2.7 implies that the 
restriction that both $G_{1}$ and $G_{2}$ be threshold in Theorem 2.6 is stronger than necessary for the union $G=G_{1} \cup G_{2}$ to be perfectly orderable. If we somehow weaken the restriction on $G_{1}$ and (or) $G_{2}$, will the union $G$ still be perfectly orderable? With this question in mind, we are going to list all the possible unions of $G_{1}$ and $G_{2}$, in which we forbid $1-3$ of these three structures $\left(P_{4}, 2 K_{2}\right.$ and $\left.C_{4}\right)$ as induced subgraphs, and study in each. case the perfect orderability of $G$.

\subsubsection{The List of Problems}

We use Table 3.1 to list our problems:

\subsubsection{Explanation of the List}

Although there are 28 different cases of two graphs $G_{1}$ and $G_{2}$ in the list, many of them can be easily eliminated by finding a counter-example showing that the union $G$ is not necessarily perfectly orderable.

\section{- Counter-example 1:}

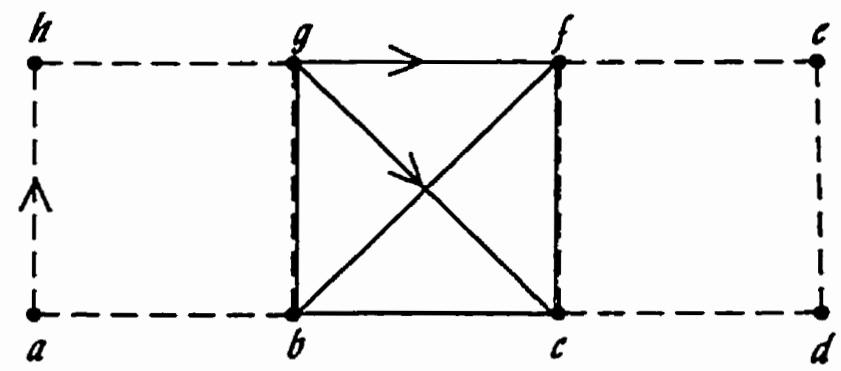

Figure 3.1

The graph in Figure 3.1 is not perfectly orderable. (If we direct $a \rightarrow h$, then two edges $g c$ and $g f$ are forced to be directed from $g \rightarrow c$ and $g \rightarrow f$, resulting in an 


\begin{tabular}{|c|c|c|c|c|}
\hline Case NO. & $\begin{array}{l}G_{1} \\
\text { contains no }\end{array}$ & $\begin{array}{l}G_{2} \\
\text { contains no }\end{array}$ & $\begin{array}{l}G_{1} \cup G_{2} \\
\text { perfectly orderable? }\end{array}$ & Reason \\
\hline 1 & $P_{4}, C_{4}, 2 K_{2}$ & $P_{4}$ & NO & Figure 3.1 \\
\hline 2 & $P_{4}, C_{4}, 2 K_{2}$ & $C_{4}$ & NO & $C_{5}$ \\
\hline 3 & $P_{4}, C_{4}, 2 K_{2}$ & $2 K_{2}$ & NO & $\overline{C_{5}}$ \\
\hline 4 & $P_{4}, C_{4}, 2 K_{2}$ & $P_{4}, C_{4}$ & YES & Theorem 2.7 \\
\hline 5 & $P_{4}, C_{4}, 2 K_{2}^{\prime}$ & $P_{4}, 2 K_{2}^{\prime}$ & $?$ & \\
\hline 6 & $P_{4}, C_{4}, 2 K_{2}$ & $C_{4}, 2 K_{2}^{\prime}$ & NO & $C_{5}$ \\
\hline 7 & $P_{4}, C_{4}, 2 K_{2}$ & $P_{4}, C_{4}, 2 K_{2}^{\prime}$ & $\overline{Y E S}$ & Theorem 2.6 \\
\hline 8 & $P_{4}, C_{4}$ & $\bar{P}_{4}$ & $\mathrm{NO}$ & case 1 \\
\hline 9 & $P_{4}, C_{4}$ & $C_{4}$ & NO & case 2 \\
\hline 10 & $P_{4}, C_{4}$ & $2 K_{2}^{\prime}$ & NO & case 3 \\
\hline 11 & $P_{4}, C_{4}$ & $P_{4}, C_{4}$ & $\mathrm{NO}$ & $C_{5}$ \\
\hline 12 & $P_{4}, C_{4}$ & $P_{4}, 2 K_{2}^{\prime}$ & $?$ & \\
\hline 13 & $P_{4}, C_{4}$ & $C_{4}, 2 K_{2}$ & NO & case 6 \\
\hline 14 & $\overline{P_{4}, 2 K_{2}}$ & $P_{4}$ & NO & case 1 \\
\hline 15 & $P_{4}, 2 K_{2}^{\prime}$ & $C_{4}$ & NO & case 2 \\
\hline 16 & $P_{4}, 2 K_{2}$ & $2 K_{2}^{\prime}$ & NO & case 3 \\
\hline 17 & $P_{4}, 2 K_{2}$ & $P_{4}, 2 K_{2}$ & $?$ & \\
\hline 18 & $P_{4}, 2 K_{2}^{\prime}$ & $C_{4}, 2 K_{2}$ & NO & case 6 \\
\hline 19 & $C_{4}, 2 K_{2}$ & $P_{4}$ & NO & case 1 \\
\hline 20 & $C_{4}, 2 K_{2}$ & $C_{4}$ & NO & case 2 \\
\hline 21 & $C_{4}, 2 K_{2}$ & $2 K_{2}$ & NO & case 3 \\
\hline 22 & $C_{4}, 2 K_{2}$ & $C_{4}, 2 K_{2}$ & NO & case 6 \\
\hline 23 & $P_{4}$ & $P_{4}$ & NO & case 1 \\
\hline 24 & $P_{4}$ & $C_{4}$ & NO & case 2 \\
\hline 25 & $P_{4}$ & $2 K_{2}$ & NO & case 3 \\
\hline 26 & $C_{4}$ & $C_{4}$ & $\mathrm{NO}$ & case 2 \\
\hline 27 & $\bar{C}_{4}$ & $2 K_{2}$ & $\mathrm{NO}$ & case 3 \\
\hline 28 & $2 K_{2}$ & $2 K_{2}$ & NO & case 3 \\
\hline
\end{tabular}

Table 3.1: The List of the Union of Two Graphs. 
impossible orientation on edge de without obstruction.)

In this Figure 3.1, we see that $G_{1}$ is a threshold graph, and $G_{2}$ is $P_{4}$-free, so it is a counter-example to case 1 in the list. Since restrictions on $G_{1}$ are weaker in cases $8,14,19$ and 23 than in case 1 , we must say "NO" to all these cases.

- Counter-example $2: C_{5}$

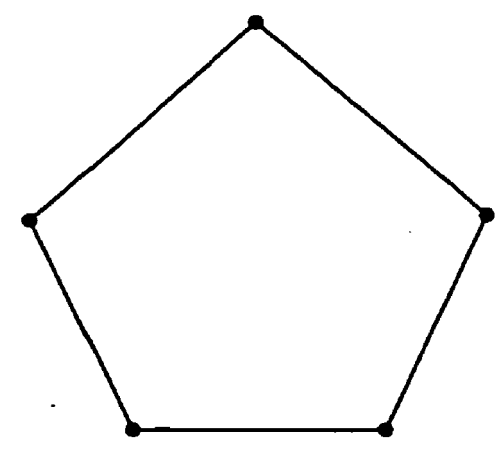

Figure 3.2: $C_{5}$.

$C_{5}$ is a well-known simple graph that is not perfectly orderable. If any of these unions in the list contains a $C_{5}$ as induced subgraph, then they are also not perfectly orderable.

In Figure 3.3, $C_{5}$ is written as the union of $G_{1}$ and $G_{2}$ such that $G_{1}$ is threshold and $G_{2}$ is $2 K_{2}$-free and $C_{4}$-free. So it is a counter-example to cases 2,3 and 6 in the list. It follows that the answers to cases $9,10,13,15,16,18,20-22,24-28$ are "NO".

In Figure 3.4, $C_{5}$ is written as the union of $G_{1}$ and $G_{2}$ such that $G_{1}$ and $G_{2}$ are both $P_{4}$-free and $C_{4}$-free. So it is a counter-example to cases 11 in the list.

Up to this point, we have given a definitive answer "YES" or "NO" to 25 out of 28 cases in the list, yet there are still 3 cases labeled with "?" that we cannot so easily answer.

Let us list again these three cases for clarity: 


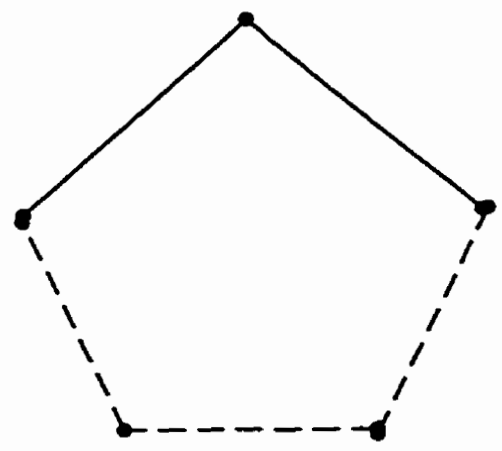

Figure 3.3: $C_{5}$.

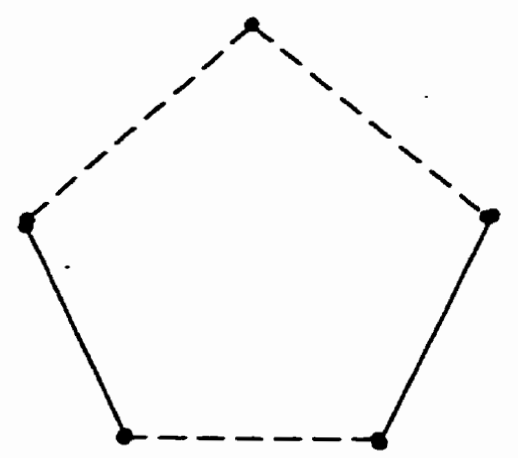

Figure 3.4: $C_{5}$.

1. Case 5: $G_{1}$ is threshold, $G_{2}$ is $P_{4}$-free and $2 K_{2}$-free;

2. Case 12: $G_{1}$ is $P_{4}$-free and $C_{4}$-free, $G_{2}$ is $P_{4}$-free and $2 K_{2}$-free;

3. Case 17: $G_{1}$ and $G_{2}$ are both $P_{4}$-free and $2 K_{2}$-free.

It is obvious that the union $G$ in case 5 is a subclass of the unions in both cases 12 and 17. 


\subsection{The Perfect Orderability of the Union of Two Graphs in Three Cases}

In this section, we shall investigate in more detail the perfect orderability of the union of two graphs in the cases $5,12,17$. First, we verify that no known minimally non-perfectly orderable graphs can be written as the union of two graphs with the properties in these three cases. Second, we verify that no known class of perfectly orderable graphs can contain such unions. Third, we pose the conjecture that such three kinds of unions are new classes of perfectly orderable graphs, and with an extra restriction that $G_{1}$ and $G_{2}$ are edge disjoint, we prove the union in case $1 \tau$. hence case 5 , is perfectly orderable.

\subsubsection{Some Minimally Non-perfectly Orderable Graphs}

In this subsection, we introduce some known minimally non-perfectly orderable graphs and show that they cannot be the unions of two graphs with the properties in case 5,12 and 17 .

\section{- Odd Hole}

Definition 3.1 An odd hole is a chordless cycle with odd length at least 5, namely $C_{2 k+1}(k \geq 2)$.

$C_{5}$ is the simplest odd hole, which we used as a counter-example to many cases in the list, as explained in the last subsection. To show that the odd hole is not a counter-example to the three unanswered cases, we prove the following observation:

Observation 3.1 Let $G_{1}$ be a graph containing no $P_{4}$, no $2 K_{2}$ as induced subgraphs, and $G_{2}$ be a graph containing no $P_{4}$ as an induced subgraph, then the union $G=$ $G_{1} \cup G_{2}$ contains neither $C_{k}(k \geq 5)$ nor $P_{k}(k \geq 8)$. 
Proof: Suppose that $G$ contains a $C_{k}(k \geq 5)$ or a $P_{k}(k \geq 8)$. Since $C_{1}$ contains no $P_{4}$ and $2 K_{2}^{\prime}$ as induced subgraphs, it is easy to see that $G_{1}$ can be of only two forms, $P_{2}$ and $P_{3}$ (Figure 3.5) in $C_{k}(k \geq 5)$ or $P_{k}(k \geq 8)$ (not considering the isolated vertices of $G_{1}$ ).

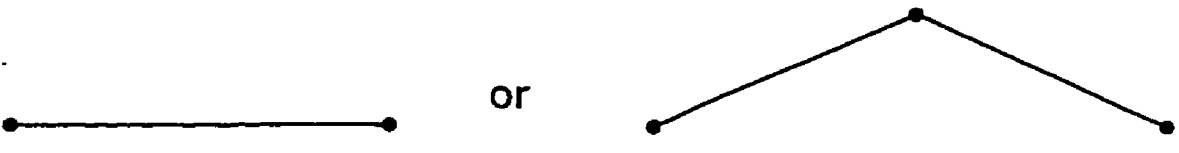

Figure 3.5: Possible $G_{1}$.

But in either case, $C_{k}-G_{1}$ or $P_{k}-G_{1}$ contains a $P_{4}$ and is a subgraph of $G_{2}$. a contradiction.

- Anti-hole

Recall that $\bar{G}$ is the complement of a graph $G$.

Definition 3.2 An anti-hole $\overline{C_{k}}$ is the complement of the cycle $C_{k}$.

Theorem 3.1 Anti-hole $\overline{C_{k}}(k \geq 5)$ is not perfectly orderable.

Proof: By contradiction. Suppose there is an admissible acyclic orientation on $\overline{C_{k}}(k \geq 5)$.

Let the vertices of an anti-hole $\overline{C_{k}}(k \geq 5)$ be denoted as $v_{0}, v_{1}, \ldots, v_{k-1}$, such that $v_{i} v_{i+1}$ is not an edge (subscript is taken modulo k).

Observation 3.2 For every edge $v_{i} v_{i+2} \in \overline{C_{k}}$, if $v_{i} \rightarrow v_{i+2}$, then $v_{i-1} \rightarrow v_{i+1}$; if $v_{i+2} \rightarrow v_{i}$, then $v_{i+3} \rightarrow v_{i+1}$.

Proof: Note that both $v_{i} v_{i+2} v_{i-1} v_{i+1}$ and $v_{i+2} v_{i} v_{i+3} v_{i+1}$ are $P_{4} \mathrm{~s}$ in $\overline{C_{k}}$. By assumption, there is no obstruction in $\overline{C_{k}}$. So if $v_{i} \rightarrow v_{i+2}$, there is a forcing $v_{i-1} \rightarrow$ 


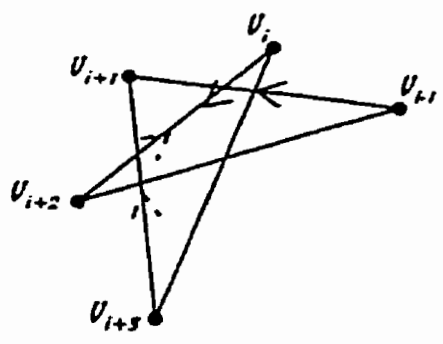

Figure 3.6

$v_{i+1}$ in the $P_{4} v_{i} v_{i+2} v_{i-1} v_{i+1}$; if $v_{i+2} \rightarrow v_{i}$, there is a forcing $v_{i+3} \rightarrow v_{i+1}$ in the $P_{4}$ $v_{i+2} v_{i} v_{i+3} v_{i+1}$ (See Figure 3.6.)

We continue the proof of the theorem. Without loss of generalit: let us assume $v_{0} v_{2}$ is directed from $v_{0}$ to $v_{2}$.

Observation 3.2 implies that if $k$ is even, $\overline{C_{k}}$ will have a directed cycle $v_{0} v_{2} c_{4} \ldots v_{k-2} v_{0}$; if $k$ is odd, $\overline{C_{k}}$ will have a directed cycle $v_{0} v_{2} v_{4} \ldots v_{k-1} v_{1} v_{3} \ldots v_{k-2} v_{0}$ (Figure $3 . \overline{1}$ ), a contradiction.

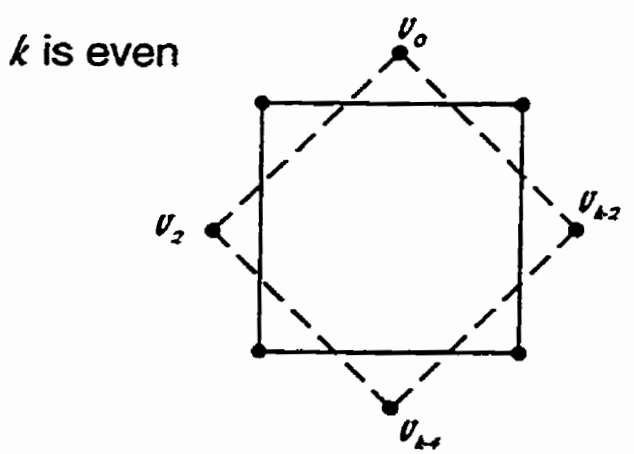

$k$ is odd

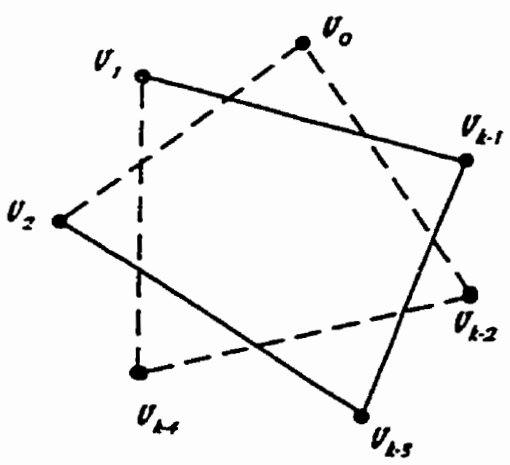

Figure 3.7: An anti-hole.

To show that an anti-hole is also not a counter-example to cases 5,12 , and 17 , we shall prove the following theorem:

Theorem 3.2 Let $G_{1}$ be a graph containing no $P_{4}$, no $2 K_{2}$ as induced subgraphs, and $G_{2}$ be a graph containing no $P_{4}$ as induced subgraph, then the union $G=G_{1} \cup G_{2}$ 
contains no $\overline{C_{k}}(k \geq 5)$.

The proof of Theorem 3.2 will be presented in section 3.3 of this chapter.

\section{- Other Examples}

Here, we give some more specific examples of minimally non-perfectly orderable graphs:

i)

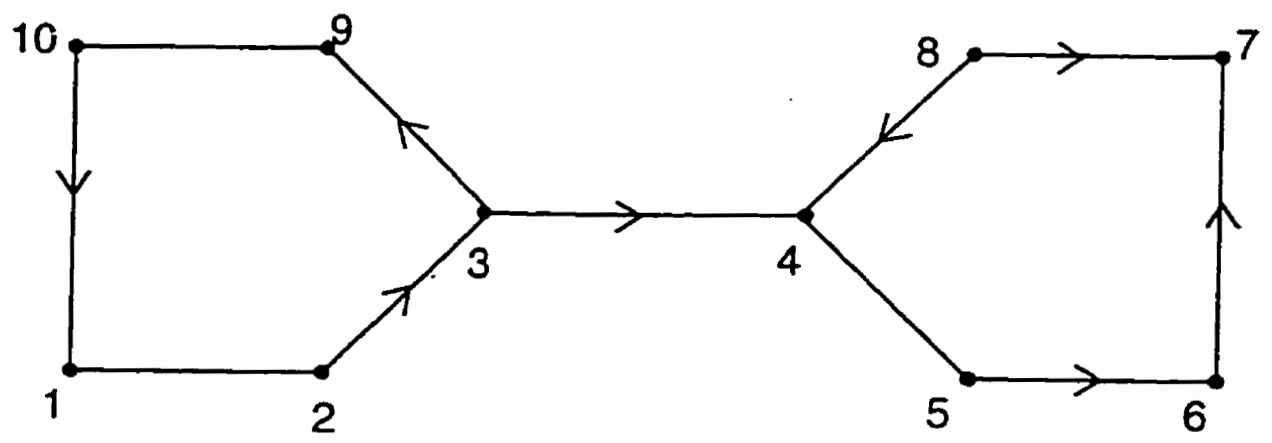

Figure 3.8: Example 1.

In Figure 3.8, without loss of generality, we may assume that $6 \rightarrow 7$. There is a chain reaction forcing $8 \rightarrow 4,3 \rightarrow 9,10 \rightarrow 1$ and $2 \rightarrow 3$, whereupon the vertices $2,3,4$ and 8 constitute an obstruction.

It is clear that the graph $G$ in Figure 3.8 contains a $P_{8}$, namely $678439(10) 1$. and using the Observation 3.1, we know that $G$ cannot be the union of two graphs in case 5,12 and 17.

ii)

The graph $G$ in Figure 3.9 is the union of a $\overline{P_{7}} 1234567$ and and a $P_{4} 3895$. Without loss of generality, we may assume $8 \rightarrow 9$. Then there is a chain reaction forcing $5 \rightarrow 7,4 \rightarrow 6,3 \rightarrow 5,2 \rightarrow 4,1 \rightarrow 3$ and $5 \rightarrow 1$ (by $8 \rightarrow 9$ ), whereupon there is a directed cycle 1351 in $G$. 


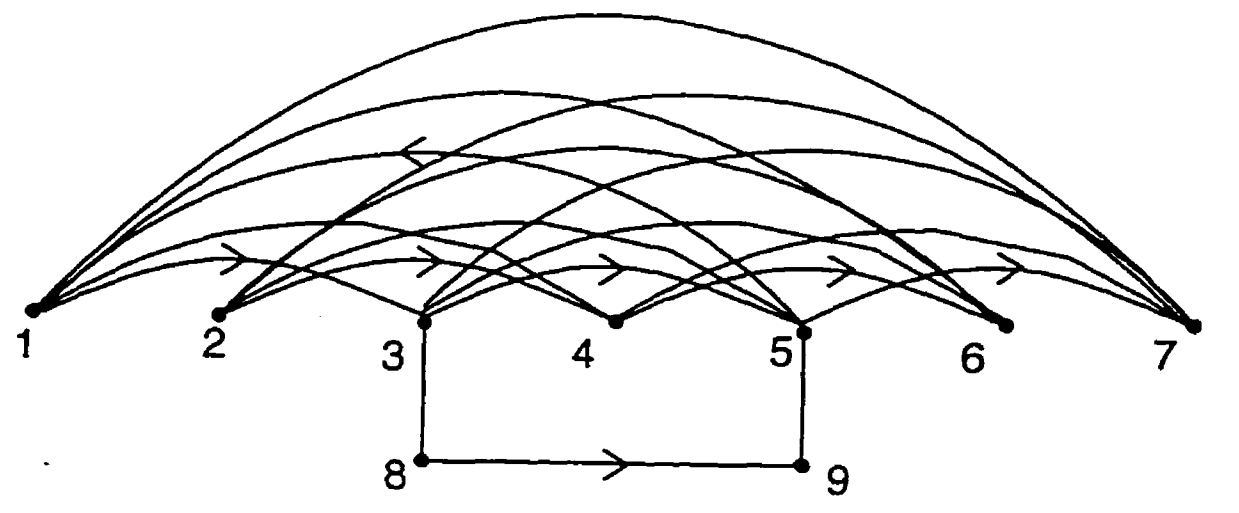

Figure 3.9: Example 2.

To show that $G$ cannot be the union of $G_{1}$ and $G_{2}$ in cases 5,12 and 17 . we shall prove the following two observations:

Observation $3.3 G$ cannot be the union of two graphs $G_{1}$ and $G_{2}$ in case 17 .

Proof: By contradiction. Suppose $G$ is the union of $G_{1}$ and $G_{2}$ in case $1 \tau$, that is $G_{1}$ and $G_{2}$ are both $P_{4}$-free and $2 K_{2}$-free.

We notice that there is a $P_{6}$, namely 895746, in $G$. Since $G_{1}$ and $G_{2}$ are both $P_{4}$-free and $2 K_{2}$-free, they can be of only two forms, $P_{2}$ or $P_{3}$, in $P_{6}$ (not considering the isolated vertices). In either case, $G_{2}$ (or $\left.G_{1}\right)=G-G_{1}$ (or $G_{2}$ ) contains a $P_{4}$, a contradiction.

Observation 3.4 $G$ cannot be the union of $G_{1}$ and $G_{2}$ in case 12 .

Proof: By contradiction. Suppose $G_{1}$ and $G_{2}$ are in case 12 , that is $G_{1}$ is $P_{4}$-free and $C_{4}$-free, $G_{2}$ is $P_{4}$-free and $2 K_{2}$-free.

Consider the $P_{6} 895746$ induced in $G$. We must have two outside edges 89 and 46 belonging to $G_{1}$ only and the middle edge 57 belonging to $G_{2}$ only. (We say that an edge $e$ belongs to $G_{i}$ only if $\epsilon \in E\left(G_{i}\right)-E\left(G_{j}\right)$ with $i \neq j$.) In the $C_{4} 8953$, since 89 is in $G_{1}$ and $G_{1}$ contains no $P_{4}$ and $C_{4}$, we can see that either i) $35,38 \in G_{2}$ only, and $59 \in G_{1}$ only, or ii) $35,95 \in G_{2}$ only, and $38 \in G_{1}$ only. Without loss of generality; 
we may assume the first case occurs. Now, if $25 \in G_{1}$, then there is a $P_{4} 259 S$ in $G_{1}$; if $25 \in G_{2}$, then there is a $P_{4} 2538$ in $G_{2}$, a contradiction.

Since the classes of unions described in cases 12 and 17 contain all unions in case 5 , the above two observations are sufficient to show $G$ cannot be the union of two graphs in cases 5 .

iii)

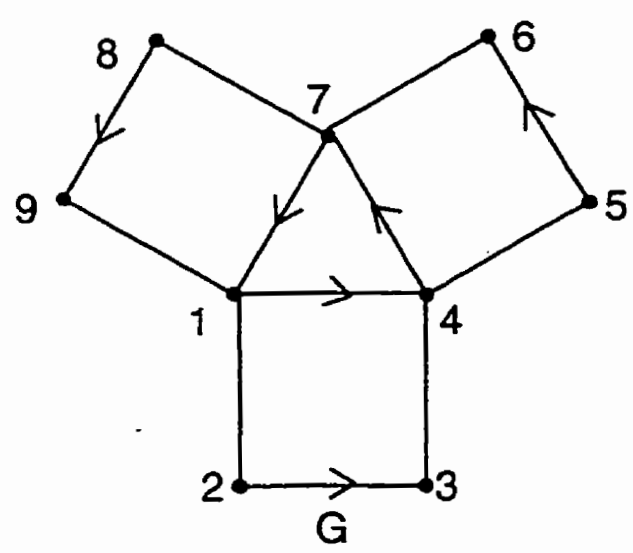

Figure 3.10: Example 3.

The graph $G$ in Figure 3.10 is a minimally non-perfectly orderable graph (the forcing on the edges of $G$ creates a directed cycle 1471).

Suppose $G=G_{1} \cup G_{2}$ as in any of cases 5,12 , or 17 . If both edges 34 and 45 belong to $G_{1}$, then consider the $P_{5} 23456$ of $G$. Since $G_{1}$ contains no $P_{4}$, the edges 23 and 56 must belong to $G_{2}$ and they form a $2 K_{2}$ in $G_{2}$, a contradiction. Otherwise, at least one of the edges 34 or 45 must be from $G_{2}$, so by symmetry assume 34 is in $G_{2}$. Similarly, at least one of the edges 67 , or 78 is in $G_{2}$. In each case, the set $\{3,4$, $6,7\}$ (or $\{3,4,7,8\}$ ) induces a $2 K_{2}$ or $P_{4}$ in $G_{2}$, a contradiction. 


\subsubsection{Comparison with Known Classes of Perfectly Order- able Graphs}

In this subsection, we will show that the union of two graphs in cases 5,12 and 17 does not belong to any known class of perfectly orderable graphs introduced in Chapter 1 .

Since the union in case 5 has more strict restrictions on $G_{1}$, it is sufficient to show that such a union does not belong to any class of perfectly orderable graphs. Next. we are going to give some examples to illustrate our conclusion.

\section{- Example 1}

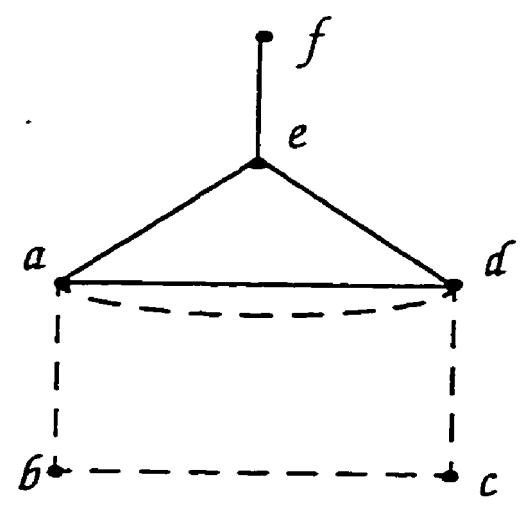

Figure 3.11: Example 1.

In figure 3.11, the graph $G$ is the union in case 5 ( $G_{1}$ consists of the solid lines, $G_{2}$ consists of the dashed lines).

i) $G$ is not a comparability graph.

It cannot be directed without constructing a semi-transitive orientation in Figure 2.5. (If we direct $b \rightarrow c$, then $d \rightarrow e$. Comparability orientations imply $f \rightarrow e, a \rightarrow e$ and $a \rightarrow b$, then we have a directed $P_{3} a \rightarrow b, b \rightarrow c$ that is not a comparability orientation.)

ii) $G$ is not a triangulated graph. 
It contains a $C_{4}$.

iii) $G$ is not a co-triangulated graph.

The two edges $e f$ and $b c$ form a $2 K_{2}$ in $G$, so there is a $C_{4}$ becf in $\bar{G}$, which is not a triangulated graph.

iv) $G$ is not a $P_{4}$-comparability graph.

Suppose that $G$ is a $P_{\mathbf{4}}$-comparability graph. Without loss of generality, we may assume $b \rightarrow c$. This implies $d \rightarrow \epsilon$ and $\epsilon \rightarrow a$. But then one of the $P_{4}$ 's feab or fedc is not of Type 3 , a contradiction.

v) $G$ is not a $P_{4}$-indifference graph.

In Figure 3.11, if $P_{4} e a b c$ is of Type 2, $P_{4}$ bcde cannot be of Type 2, otherwise there will be a directed cycle $b c d e a b$.

vi) $G$ is not a Raspail graph.

$G$ contains a $a$ house which is a forbidden subgraph in Raspail graphs.

vii) $G$ is not a Welsh-Powell perfect nor a Matula perfect graph.

A house is also a forbidden structure in both Welsh-Powell and Matula perfect graphs.

viii) $G$ is not a union of two threshold graphs.

This is obvious.

\section{- Example 2}

The graph $G$ in Figure 3.12 (a) is the union of two threshold graphs $G_{1}$ and $G_{2}$, with $G_{1}$ consisting of the edges $a b, a c, b c$ and $G_{2}$ consisting of the remaining edges.

Claim 3.1 $G$ is not an intersection of two threshold graphs.

Proof: Suppose $G$ is the intersection of two threshold graphs $G_{1}, G_{2}$.

Observation 3.5 Let $e_{1}, e_{2}$ be two edges that induce a $2 K_{2}^{\prime}$ in $G$. Then some edge $e_{i}(i=1,2)$ must have one of its endpoints being adjacent to the two endpoints of $e_{j}$ 


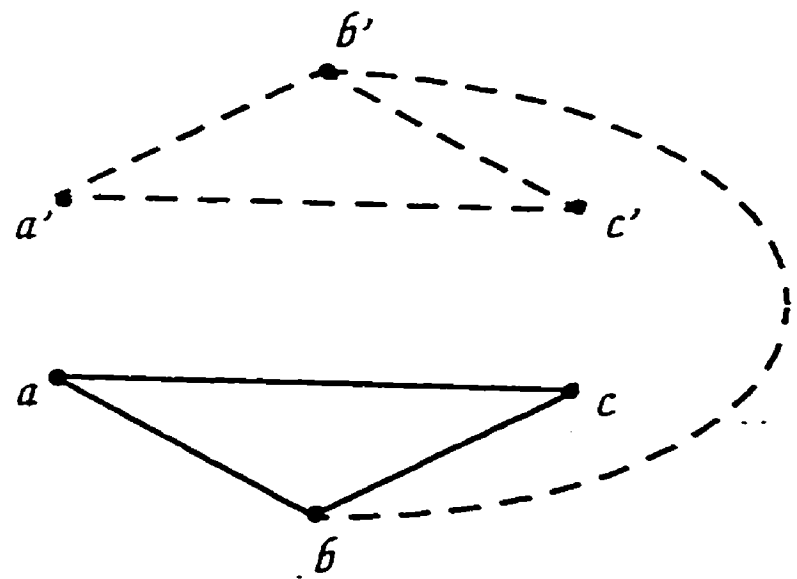

(a)

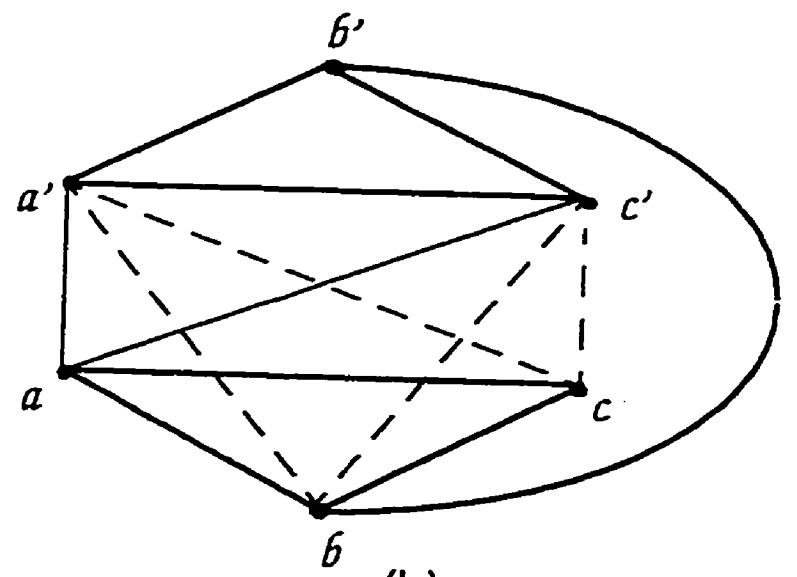

(b)

Figure 3.12

$(j \neq i)$ in $G_{1}$ and the other endpoint being adjacent to the two endpoints of $e_{j}$ in $G_{2}$ (Figure 3.13).

(In Figures 3.13 and $3.12(\mathrm{~b})$, the thick lines denote edges in both $G_{1}$ and $G_{2}$, the solid lines denote edges in $G_{1}$, and the dashed lines denote edges in $G_{2}$.)

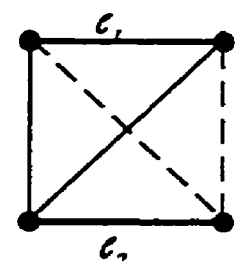

Figure 3.13

Consider the $2 K_{2}$ formed by $\left\{a^{\prime}, c^{\prime}, a, c\right\}$. By Observation 3.5, we may assume that $a a^{\prime}, a c^{\prime} \in E\left(G_{1}\right)$ and $c a^{\prime}, c c^{\prime} \in E\left(G_{2}\right)$. Now consider the $2 K_{2}$ formed by $\left\{a, b, a^{\prime}, c^{\prime}\right\}$, Observation 3.5 implies that $b a^{\prime}, b c^{\prime} \in E\left(G_{2}\right.$. But the set $\left\{a^{\prime}, c^{\prime}, b, c\right\}$ induces a $2 K_{2}^{\prime}$ in $G_{1}$, a contradiction.

We conclude that there exists a union in cases 5,12 , and 17 that does not belong to the class of intersections of two threshold graphs.

\section{- Example 3}




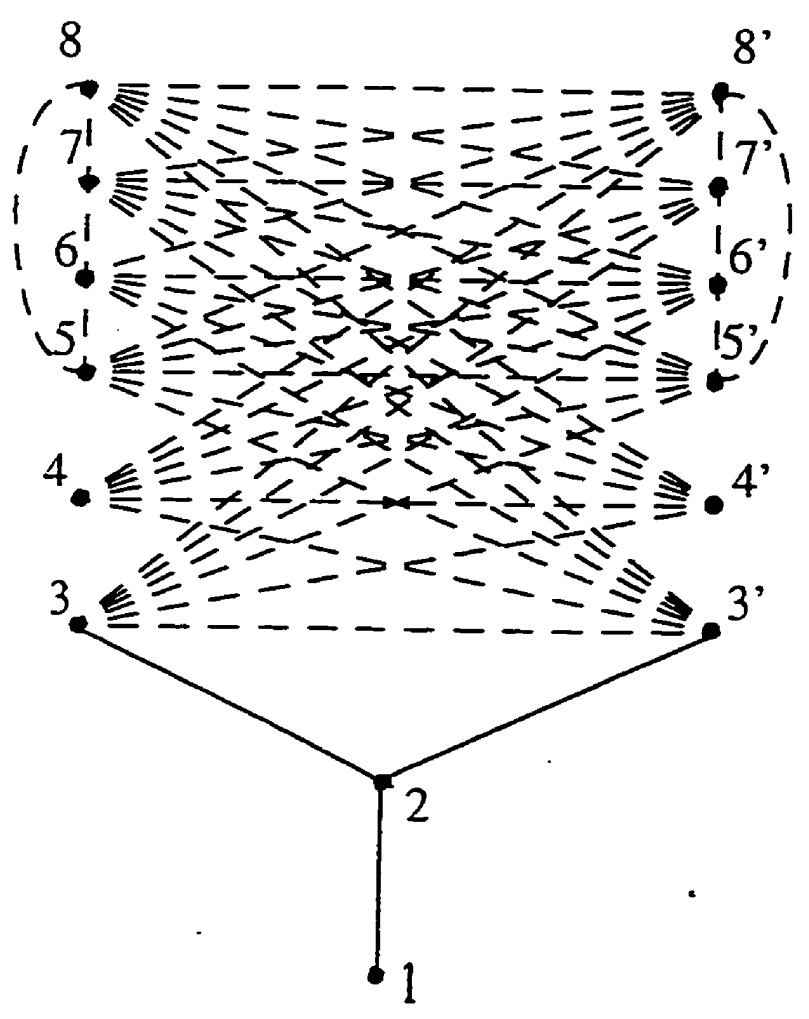

Figure 3.14: A non-D-graph.

The graph $G$ in Figure 3.14 is a union of $G_{1}$ (in solid line) and $G_{2}$ (in dashed line). It is easy to verify that $G_{1}$ is threshold, and $G_{2}$ is $P_{4}$-free and $2 K_{2}$-free.

The graph $G$

i) has Dilworth number greater than 3.

Let $S=\{5,6,3,1\}$. It is easy to verify that the vertices in $S$ are pairwise incomparable, so the Dilworth number of $G$ is at least 4 .

ii) is not a $D$-graph.

Recall that a $d$-vertex $w$ is such a vertex that for every edge $a b$ with $\{a, b\} \cap N(w)=$ $\emptyset, a$ and $b$ are comparable. There is no $d$-vertex in $G$ (for vertices $1,2,3$ and 4 , we have that 5 and 6 are incomparable; for vertices $3^{\prime}$ and $4^{\prime}$, we have that $5^{\prime}$ and $6^{\prime}$ are incomparable; for vertices $5,6,7$ and 8 (respectively $5^{\prime}, 6^{\prime}, 7^{\prime}$, and $8^{\prime}$ ), we have that 3 $\left(3^{\prime}\right)$ and 1 are incomparable). 


\section{- Example 4}

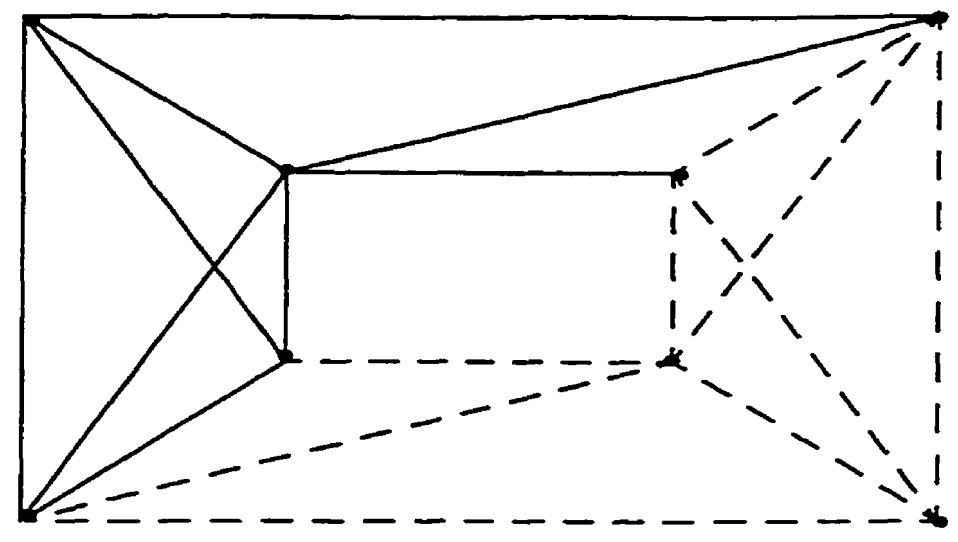

Figure 3.15

The graph $G$ shown in Figure 3.15 is a union of two threshold graphs. The reader may easily check that each vertex is the endpoint of some $P_{4}$ and the midpoint of some other $P_{4}$. Thus $G$ is not brittle (and therefore not $P_{4}$-simplicial).

The above four examples show that no known class of perfectly orderable graphs contains all unions of two graphs in case 5 , or 12 , or 17 .

\subsubsection{Conjectures}

Since we are unable to find a non perfectly orderable graph that can be written as the union of two graphs in case 5 , or 12 , or 17 , we would like to propose the following conjecture:

Conjecture 1 If $G$ is the union of two graphs $G_{1}$ and $G_{2}$ satisfying any of the following conditions:

1. $G_{1}$ is threshold, $G_{2}$ is $P_{4}$-free and $2 K_{2}-$ free;

2. $G_{1}$ is $P_{4}$-free and $C_{4}$-free, $G_{2}$ is $P_{4}$-free and $2 K_{2}$-free; 
3. $G_{1}$ and $G_{2}$ are both $P_{4}$-free and $2 K_{2}$-free.

then $G$ is perfectly orderable.

If Conjecture 1 is true, then the three classes of graphs described by it would form new classes of perfectly orderable graphs (by the results in subsection 3.2.2).

Although we have not found a way to prove this conjecture, we do prove. jointly with Hoàng, the following theorem by adding the extra constraint that $G_{1}$ and $G_{2}$ are edge-disjoint for case 3.

Theorem 3.3 If $G_{1}$ and $G_{2}$ are both $P_{4}$-free and $2 K_{2}$-free, and $G_{1}$ and $G_{2}$ are edge disjoint, then $G=G_{1} \cup G_{2}$ is perfectly orderable.

The proof of this theorem is given in the next section.

\subsection{Proof of the Theorems}

Theorem 3.4 Let $G_{1}$ be a graph containing no $P_{4}$, no $2 K_{2}$ as induced subgraphs, and $G_{2}$ be a graph containing no $P_{4}$ as induced subgraph, then $G=G_{1} \cup G_{2}$ contains no $\overline{C_{k}}(k \geq 5)$.

Proof: By contradiction. Suppose $G$ contains $\overline{C_{k}}(k \geq 5)$. Number the vertices of $\overline{C_{k}}$ as $v_{0}, v_{1} \ldots v_{k-1}$, such that $v_{i} v_{i+1}$ is not an edge (subscript is taken modulo $k$ ). Since it is easy to verify that the Theorem is true for $k=5$, we may assume that $k>5$.

Before making any further argument, we are going to introduce the following observation, which is frequently used in the proof (whenever we say an edge $e$ is in $G_{l}(l=1,2)$ only, we mean $\left.e \in E\left(G_{l}\right)-E\left(G_{t}\right), t \neq l\right)$ :

Observation 3.6 In any induced $C_{4}$ of $\overline{C_{k}}(k \geq 5)$, namely abcd, if $a b$ is in $G_{1}$ only, and bc is in $G_{2}$ only, then ad is in $G_{1}$ only, and $c d$ is in $G_{2}$ only (Figure 3.16). 


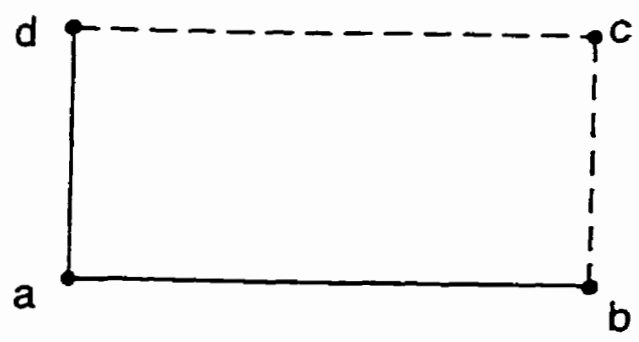

Figure 3.16

Claim 3.2 For any four consecutive vertices $v_{i}, v_{i+1}, v_{i+2}, v_{i+3}(i=0.1 \ldots . . k-1)$. the two edges $v_{i} v_{i+2}$ and $v_{i+1} v_{i+3}$ cannot be in the same $G_{l}(l=1.2)$.

Proof: Suppose $v_{i} v_{i+2}$ and $v_{i+1} v_{i+3}$ are in the same $C_{i l}$. we need to consider only four cases:

1. They are both in $G_{1}$ only;

2. They are both in $G_{2}$ only;

3. One is in both $G_{1}$ and $G_{2}$, the other one is in $G_{l}(l=1,2)$ only;

4. They are both in $G_{1}$ and $G_{2}$.

Since $G_{1}$ contains no $P_{4}$ and $2 K_{2}^{\prime}$, it is obvious that case 1 and case 4 are not possible. We are now going to show that case 2 and case 3 are not possible either.

case 2: Suppose $v_{i} v_{i+2}$ and $v_{i+1} v_{i+3}$ are in $G_{2}$ only. Since $v_{i+1} v_{i+3} v_{i} v_{i+2}$ is a $P_{4}$ of $G, v_{i} v_{i+3}$ must be in $G_{1}$ only. Consider the $C_{4} v_{i-1} v_{i+2} v_{i} v_{i+3}$ : using Observation 3.6, we have $v_{i-1} v_{i+2}$ belonging to $G_{2}$ only and $v_{i+3} v_{i-1}$ belonging to $G_{1}$ only. Now $v_{i-1} v_{i+1}$ cannot belong to either $G_{1}$ or $G_{2}$ (if $v_{i-1} v_{i+1}$ is in $G_{1}$ then $v_{i+1} v_{i-1} v_{i+3} v_{i}$ is a $P_{4}$ in $G_{1}$; if $v_{i-1} v_{i+1}$ is in $G_{2}$ then $v_{i+1} v_{i-1} v_{i+2} v_{i}$ is a $P_{4}$ in $G_{2}$ ), a contradiction (see Figure 3.1 $i$.)

case 3: Suppose $v_{i} v_{i+2}$ is in both $G_{1}$ and $G_{2}, v_{i+1} v_{i+3}$ is in $G_{l}(l=1,2)$ only. 


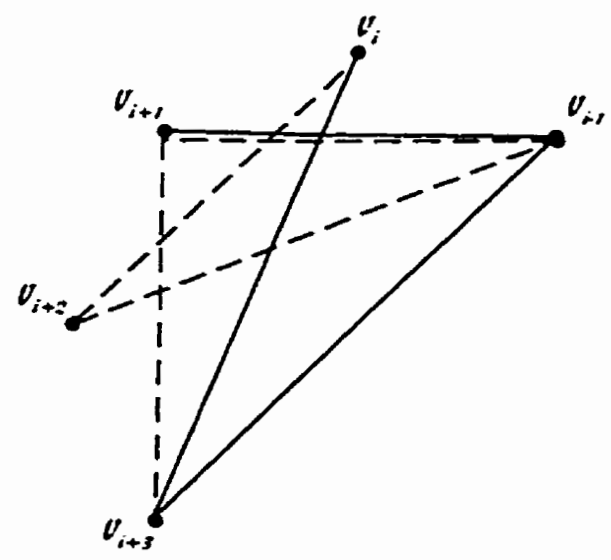

Figure 3.17

Since $G_{1}$ contains no $P_{4}$ and $2 K_{2}, v_{i+1} v_{i+3}$ cannot be in $G_{1}$ (otherwise, the set $\left\{v_{i}, v_{i+1}, v_{i+2}, v_{i+3}\right\}$ induces a $P_{4}$.or $2 K_{2}^{\prime}$ in $\left.G_{1}\right)$, and must be in $G_{2}$ only, and $v_{i} v_{i+3}$ is in $G_{1}$ only.

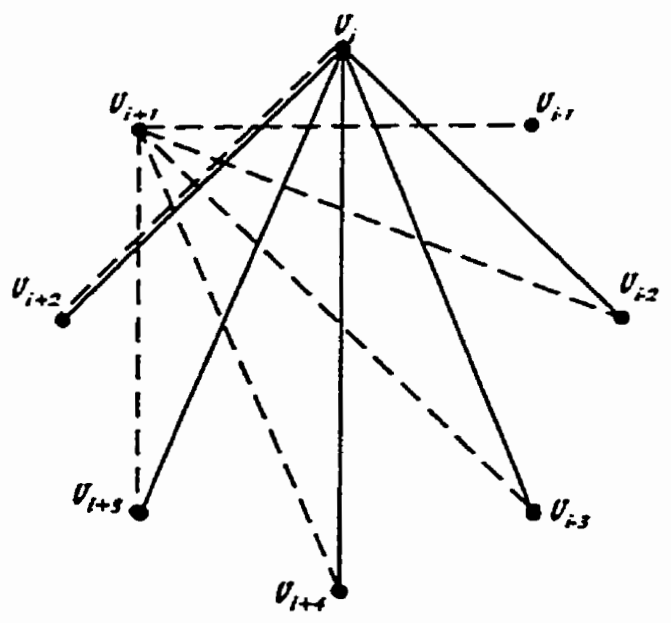

Figure 3.18

Consider the $C_{4} v_{i} v_{i+3} v_{i+1} v_{i+4}$ : Observation 3.6 implies that $v_{i+4} v_{i+1}$ is in $G_{2}$ only, and $v_{i} v_{i+4}$ is in $G_{1}$ only. For $j=i+4, i+5, \ldots, k-1,0,1, \ldots, i-3$, by considering the $C_{4} v_{i} v_{j} v_{i+1} v_{j+1}$, we see that every edge $v_{i} v_{j+1}$ is in $G_{1}$ only and every edge $v_{i+1} v_{j+1}$ is in $G_{2}$ only. Consider the $P_{4} v_{i} v_{i-2} v_{i+1} v_{i-1}$ : with $v_{i+1} v_{i-2}$ belonging to $G_{2}$ only and 
$v_{i-2} v_{i}$ belonging to $G_{1}$ only, it is obvious that $v_{i+1} v_{i-1}$ is in $G_{2}$ only. So all the edges going out from $v_{i}$ (except for $v_{i} v_{i+2}$ ) are in $G_{1}$ only, and all the edges going out from $v_{i+1}$ are in $G_{2}$ only (see Figure 3.18.)

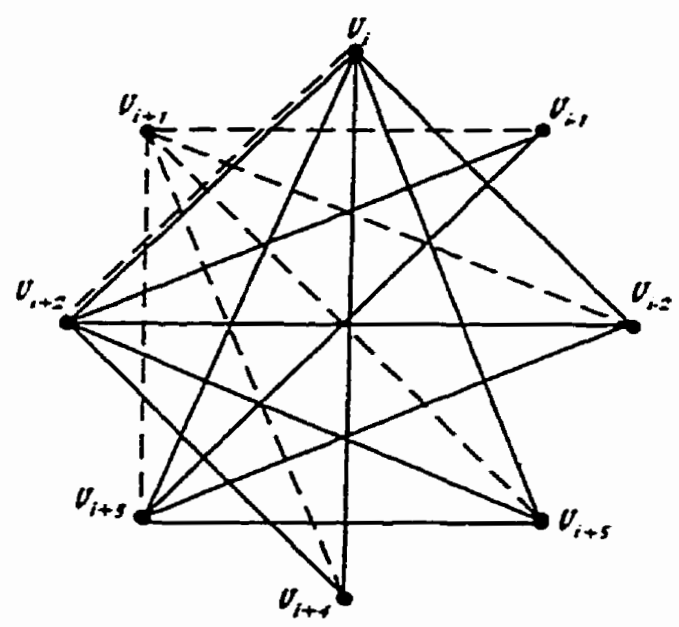

Figure 3.19

Consider the $P_{4} v_{i+3} v_{i+1} v_{i+4} v_{i+2}$ : with both edges $v_{i+1} v_{i+3}$ and $v_{i+1} v_{i+4}$ belonging to $G_{2}$ only, it is clear that $v_{i+2} v_{i+4}$ is in $G_{1}$ only. For $j=i+4, \ldots, k-1,0,1, \ldots, i-2$, consider the $C_{4} v_{i+1} v_{j} v_{i+2} v_{j+1}$, where $v_{i+1} v_{j}$ and $v_{i+1} v_{j+1}$ are in $G_{2}$ only and $v_{i+2} v_{j}$ is in $G_{1}$ only, we have that every edge $v_{i+2} v_{j+1}$ is in $G_{1}$ only. For $j=i, i-1, \ldots, 0, k-$ $1, \ldots, i+4$, by considering the $C_{4} v_{i+3} v_{j} v_{i+2} v_{j-1}$, where $v_{i+3} v_{j}, v_{j} v_{i+2}$ and $v_{i+2} v_{j-1}$ are in $G_{1}$, we see that $v_{i+3} v_{j-1}$ is in $G_{1}$. Now consider the $P_{4} v_{i+3} v_{i+5} v_{i+2} v_{i+4}$ : we see that all three edges belong to $G_{1}$, a contradiction. This completes the proof of Claim 3.2 (see Figure 3.19.)

Observation 3.7 In any induced $C_{4} a b c d$ of $\overline{C_{k}}$, if $a b$ is in $G_{1}$ only, and ad and bc are in $G_{2}$, then ad and bc must belong to $G_{1}$.

Proof: It is clear that if $a d$ and $b c$ are both in $G_{2}$ only, we either have a $2 K_{2} a b$ and $c d$ in $G_{1}$, or a $P_{4} a d c b$ in $G_{2}$, a contradiction. Without loss of generality, assume ad belongs to $G_{1}$ and $b c$ does not. Then either $a d c b$ is a $P_{4}$ in $G_{2}$ or badc is a $P_{4}$ in $G_{1}$. 


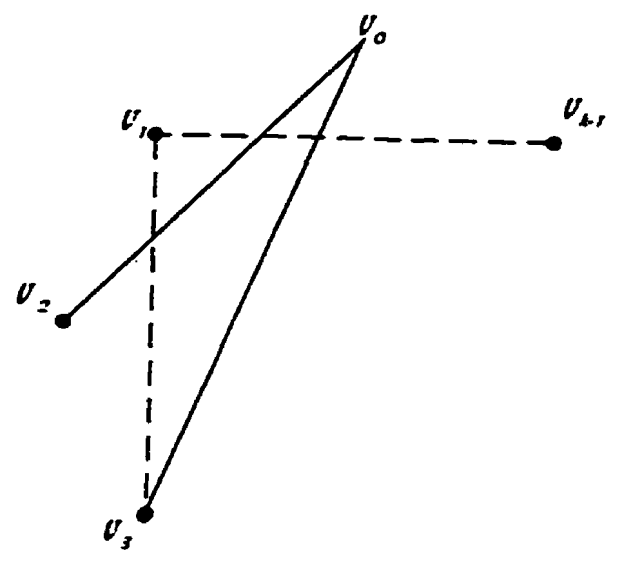

Figure 3.20

Now, we continue the proof of the theorem.

By Claim 3.2 we may assume. without loss of generality, that $v_{0} v_{2}$ is in $G_{1}$ only (if $v_{0} v_{2}$ is in $G_{2}$ then $v_{1} v_{3}$ is in $\dot{G}_{1}$ only, by renumbering the vertices of $\overline{C_{k}}$, we could arrive at the same conclusion). Again, Claim 3.2 implies that $v_{1} v_{k-1}$ and $v_{1} v_{3}$ are in $G_{2}$ only. Consider the $C_{4} v_{0} v_{2} v_{k-1} v_{3}$ in $G$. By Observation 3.7, one of the following two cases must occur: (i) either $v_{0} v_{3}$ belongs to $G_{1}$ only or $v_{2} v_{k-1}$ belongs to $G_{1}$ only, or (ii) both $v_{0} v_{3}$ and $v_{2} v_{k-1}$ belong to $G_{1}$ and $G_{2}$. Suppose that case (ii) occurs. Then we have $v_{3} v_{k-1}$ belonging to $G_{1}$ only; for otherwise $v_{0} v_{3} v_{k-1} v_{2}$ is a $P_{4}$ in $G_{2}$. But now $v_{3} v_{1} v_{k-1} v_{2}$ is a $P_{4}$ in $G_{2}$. Thus we know that case (i) must occur. Without loss of generality, we may assume that $v_{0} v_{3}$ is in $G_{1}$ only (see Figure 3.20).

For $j=3,4, \ldots, k-3$, consider the $C_{4} v_{0} v_{j} v_{1} v_{j+1}$ : with $v_{0} v_{j}$ belonging to $G_{1}$ only and $v_{1} v_{j}$ belonging to $G_{2}$ only, by Observation 3.6 we have $v_{0} v_{j+1}$ belonging to $G_{1}$ only and $v_{1} v_{j+1}$ belonging to $G_{2}$ only (see Figure 3.21 .)

Consider the $P_{4} v_{3} v_{1} v_{4} v_{2}$ : with two edges $v_{3} v_{1}$ and $v_{1} v_{4}$ belonging to $G_{2}$ only, we must have $v_{2} v_{4}$ belonging to $G_{1}$ only. For $j=4,5, \ldots, k-2$, by considering the $C_{4}$ $v_{1} v_{j} v_{2} v_{j+1}$, with both edges $v_{j} v_{1}$ and $v_{1} v_{j+1}$ belonging to $G_{2}$ only, and $v_{2} v_{j}$ belonging to $G_{1}$ only, we have $v_{2} v_{j+1}$ belonging to $G_{1}$ only (see Figure 3.22 .) 


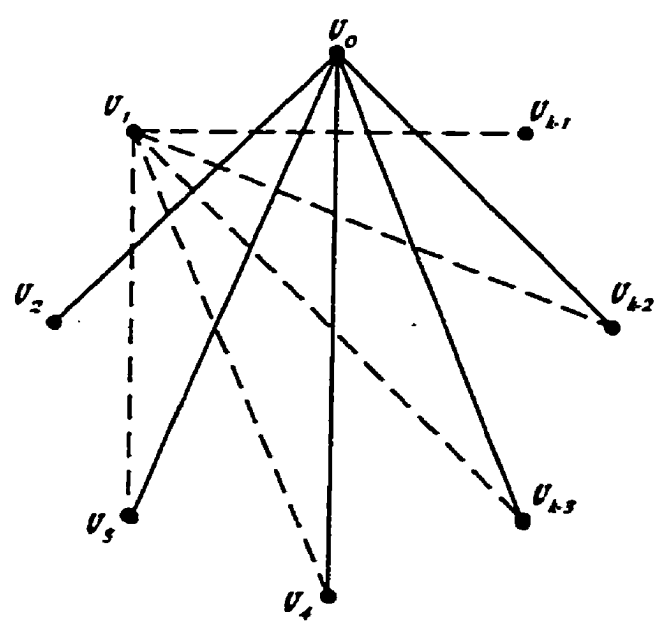

Figure 3.21

Consider the $C_{4} v_{0} v_{2} v_{k-1} v_{3}:$ with $v_{3} v_{0}, v_{0} v_{2}$, and $v_{2} v_{k-1}$ belonging to $G_{1}$, we have $v_{3} v_{k-1}$ belonging to $G_{1}$. For $j=k-2, k-3, \ldots, 5$, considering the $C_{4} v_{2} v_{j} v_{3} v_{j-1}$ : with three edges $v_{3} v_{j-1}, v_{j-1} v_{2}$ and $v_{2} v_{j}$ belonging to $G_{1}$, we must have $v_{3} v_{j}$ belonging to $G_{1}$ as well. Now, look at the $P_{4} v_{3} v_{5} v_{2} v_{4}$, all three edges of $P_{4}$ are in $G_{1}$, a contradiction to the definition of $G_{1}$ (see Figure 3.23.)

Before we start the proof of Theorem 3.3, we restate it:

Theorem 3.3 If $G_{1}$ and $G_{2}$ are both $P_{4}$-free and $2 K_{2}$-free, and $G_{1}$ and $G_{2}$ are edge disjoint, then $G=G_{1} \cup G_{2}$ is perfectly orderable.

Proof: Let $G_{1}, G_{2}$ and $G$ be as specified in the statement of the Theorem.

Claim 3.3 For every edge $a b \in E\left(G_{1}\right)$ (respectively $a b \in E\left(G_{2}\right)$ ), the two vertices $a$, $b$ must be comparable in $G_{2}$ (respectively $G_{1}$ ).

Proof: Suppose $a b \in E\left(G_{1}\right)$ and $a, b$ are not comparable in $G_{2}$. It follows that there exist two edges $a d \in E\left(G_{2}\right), b c \in E\left(G_{2}\right)$, such that $a c \notin E\left(G_{2}\right)$ and $b d \notin E\left(G_{2}\right)$. Then there will be either a $2 K_{2}$ or $P_{4}$ in $G_{2}$, which is a contradiction (see Figure 3.24.) 


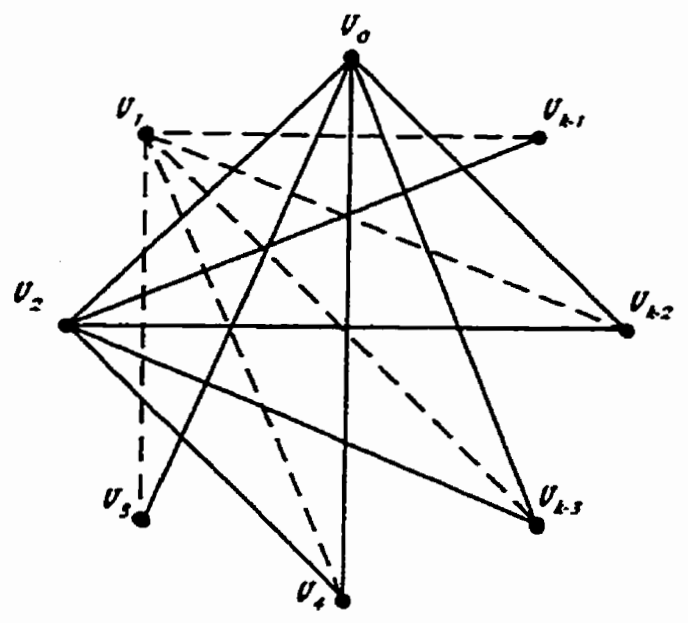

Figure 3.22

Now define a partial orientation on $G$ (we say that $a$ strictly dominates $b$ if $a$ dominates $b$ but $b$ does not dominate $a$ ).

1. $\forall a b \in E\left(G_{1}\right)$, direct $a \rightarrow b$ if and only if $a$ strictly dominates $b$ in $G_{2}$;

2. $\forall c d \in E\left(G_{2}\right)$, direct $c \rightarrow d$ if and only if $c$ strictly dominates $d$ in $G_{1}$.

Claim 3.4 $G$ has no obstruction under the partial orientation.

Proof: Since $G_{1}$ and $G_{2}$ are $P_{4}$-free and $2 K_{2}$-free, the $P_{4} a b c d$ in $G$, if there is any, must be of the following two forms (see Figure 3.25):

1. $a b, b c \in E\left(G_{1}\right), c d \in E\left(G_{2}\right)$,

2. $a b \in E\left(G_{1}\right), b c, c d \in E\left(G_{2}\right)$.

By definition of the partial orientation, we have $c \rightarrow d$ in 1) and $b \rightarrow a$ in 2). In either case, there is no obstruction.

Claim 3.5 The partial orientation creates no cycle in $G$. 


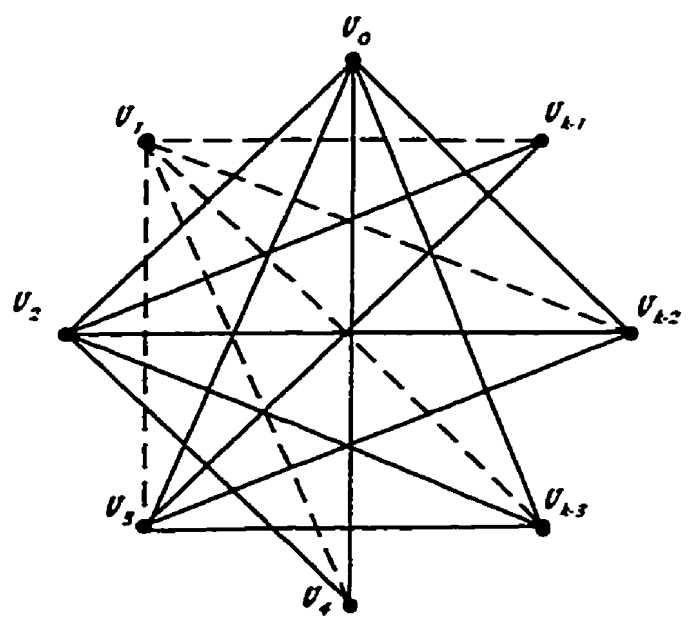

Figure 3.23

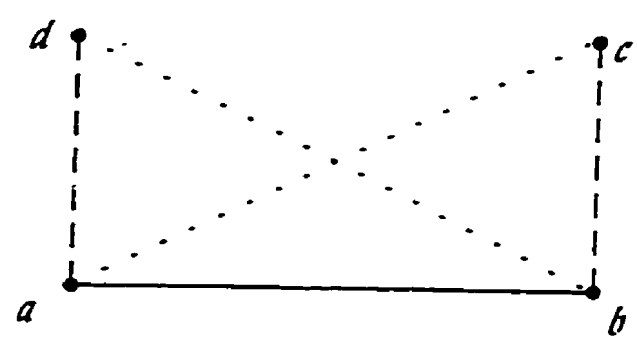

Figure 3.24

Proof: Clearly, there are no cycles in the same $G_{l}(l=1,2)$ since the domination relation is transitive.

Now we are going to show there is no mixed cycle, the cycle made up of directed edges in $G_{1}$ and $G_{2}$.

Observation 3.8 If $\overrightarrow{a b} \in E\left(G_{1}\right)$ (respectively, $\overrightarrow{a b} \in E\left(G_{2}\right)$ ) and $\overrightarrow{b c} \in E\left(G_{2}\right)$ (respectively, $\overrightarrow{b c} \in E\left(G_{1}\right)$ ), then there must be $\overrightarrow{a c} \in E\left(G_{2}\right)$ (respectively, $\overrightarrow{a c} \in E\left(G_{1}\right)$ ).

Proof: $\overrightarrow{a b} \in E\left(G_{1}\right)$ implies $a$ strictly dominates $b$ in $G_{2}$. Since $\overrightarrow{b c} \in E\left(G_{2}\right)$, we have $a c \in E\left(G_{2}\right)$, and by Claim 3.3, $a$ and $c$ are comparable in $G_{1}$. Since $a b \in E\left(G_{1}\right)$ and $c b \notin E\left(G_{1}\right), a$ strictly dominates $c$ and there is an orientation from $a$ to $c$ (see Figure 3.26.) 
1)
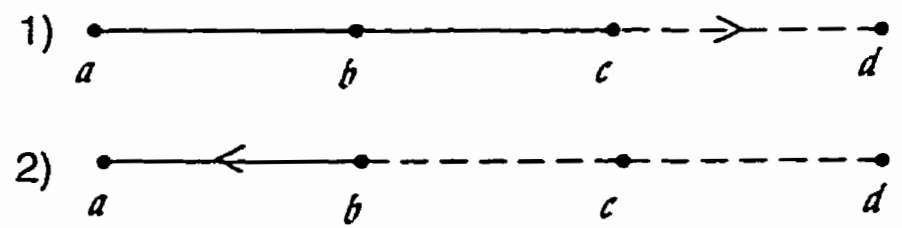

Figure 3.25: Two possible $P_{4}$.

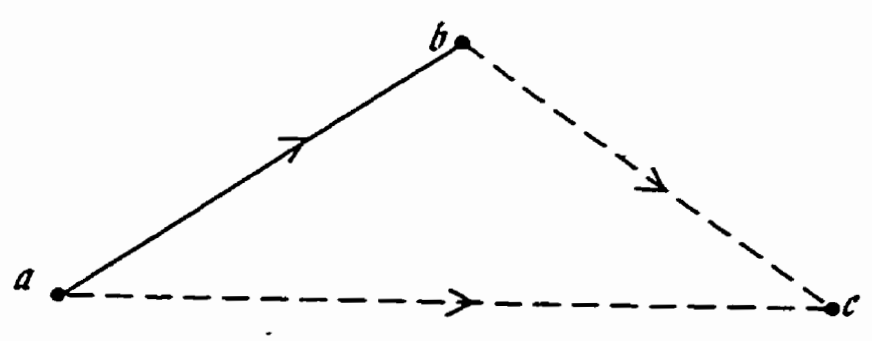

Figure 3.26

Observation 3.8 implies that there is no mixed cycle on three vertices.

Suppose there is a cycle in $G$, then it must be a mixed cycle. Let $C$ be a cycle $v_{0} v_{1} \ldots v_{l-1}$ with smallest length $l$. Select $v_{i}(i \in\{0,1, \ldots, l-1\})$, such that $\overrightarrow{v_{i-1}} v_{i} \in$ $E\left(G_{1}\right)$, and $v_{i} \vec{v}_{i+1} \in E\left(G_{2}\right)$. By the Observation 3.8, we have $v_{i-1} \vec{v}_{i+1} \in E\left(G_{2}\right)$. Then there is a new cycle $v_{0} v_{1} \ldots v_{i-1} v_{i+1} \ldots v_{l-1}$ of length $l-1$, contradicting with the assumption that $C$ is the smallest cycle.

Based on this acyclic partial orientation, we can easily construct a linear order < on $G$ by the following procedure:

1. $i \leftarrow 1, H \leftarrow G$;

2. Find a vertex $v$ in $H$ with indegree 0 and assign the order $i$ to $v$; if no such a $v$ exists, order the remaining vertices in $H$ randomly and then exit;

3. $i \leftarrow i+1, H \leftarrow H-v$, if $H \neq \emptyset$ goto step 2 . 
Such an order $<$ is sure to be obstruction-free, therefore, $G$ is perfectly orderable.

\subsection{Discussion of the Proof of Theorem 3.3}

In the proof of Theorem 3.3, we first impose a partial orientation on the edges of $G$ according to a certain rule, which guarantees the orientation to be unique on each edge, obstruction-free and acyclic, later construct a perfect linear order $<$ on the vertices of $G$ based on this partial orientation, thus get the conclusion that $G$ is perfectly orderable. The key point is how to find such an effective rule for orienting edges. We found it because we noticed the fact that in the two possible $P_{4} s$ in Figure 3.25 , if we direct the edges in $G_{1}$ (respectively $G_{2}$ ) according to the strict domination in $G_{2}$ (respectively $G_{1}$ ), it guarantees there is at least one wing of $P_{4}$ going out from the joint to the tip, preventing an obstruction. With the conditions provided in Theorem 3.3, we are able to show that such a rule is good in the since it can produce a perfect order on $G$. Here, the constraint that $G_{1}$ and $G_{2}$ are edge-disjoint is important, otherwise such a rule will not work, even for the union of two threshold graphs. In the graph of Figure 3.27, a union of two threshold graphs (one in solid lines and one in dashed lines), this strict domination rule causes a directed cycle.

Realizing the effect of such a constraint on the rule, we naturally ask this question: can a union $G$ of two threshold graphs $G_{1}$ and $G_{2}$ be decomposed into two edge-disjoint graphs $G_{1}^{\prime}$ and $G_{2}^{\prime}$, such that $G_{1}^{\prime}$ and $G_{2}^{\prime}$ are $P_{4}$-free and $2 K_{2}$-free? Unfortunately, a counter-example is found in Figure 3.28 to give a "NO" answer to the question above.

The graph in Figure 3.28 is a union of two threshold graphs with a common edge in both $G_{1}$ and $G_{2}$. Since both $G_{1}^{\prime}$ and $G_{2}^{\prime}$ are $P_{4}$-free and $2 K_{2}^{\prime}$-free, if we put edge ef in $G_{1}^{\prime}$, all the edges in $G_{2}$ except $a b$ must be put in $G_{2}^{\prime}$; similarly, if $e^{\prime} f^{\prime}$ is in $G_{2}^{\prime}$, 


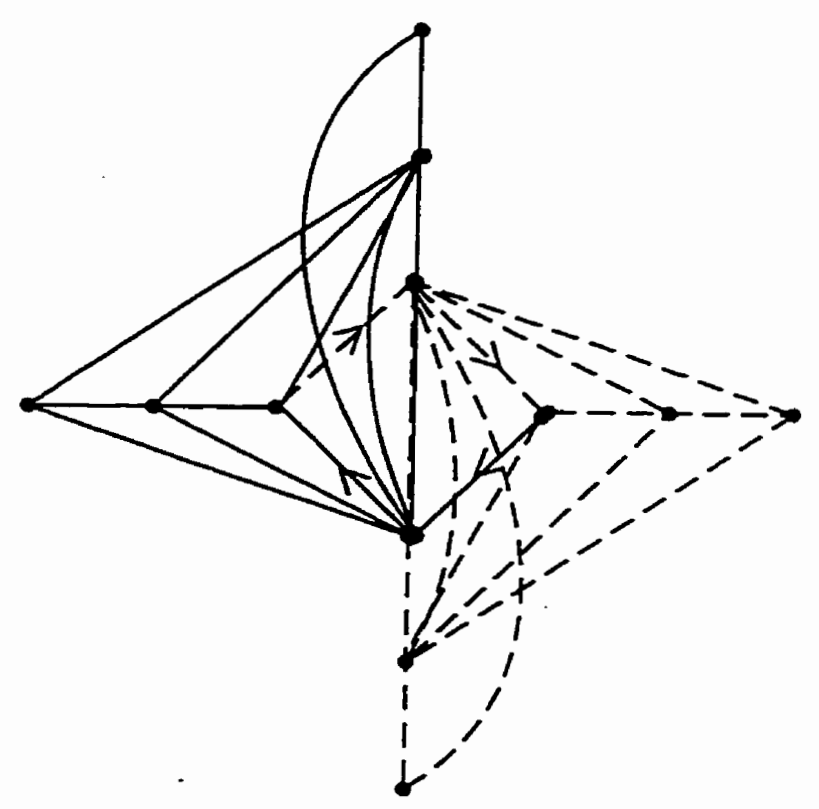

Figure 3.27: A counter-example to the rule.

all the edges in $G_{1}$ except $a b$ must be in $G_{1}^{\prime}$. If $a b$ is not in $G_{2}^{\prime}$, there will be a $P_{4}$ $a c^{\prime} b d^{\prime}$ in $G_{2}^{\prime} ;$ if $a b$ is not in $G_{1}^{\prime}$, there will be a $P_{4} d a c b$ in $G_{1}^{\prime}$, a contradiction. 


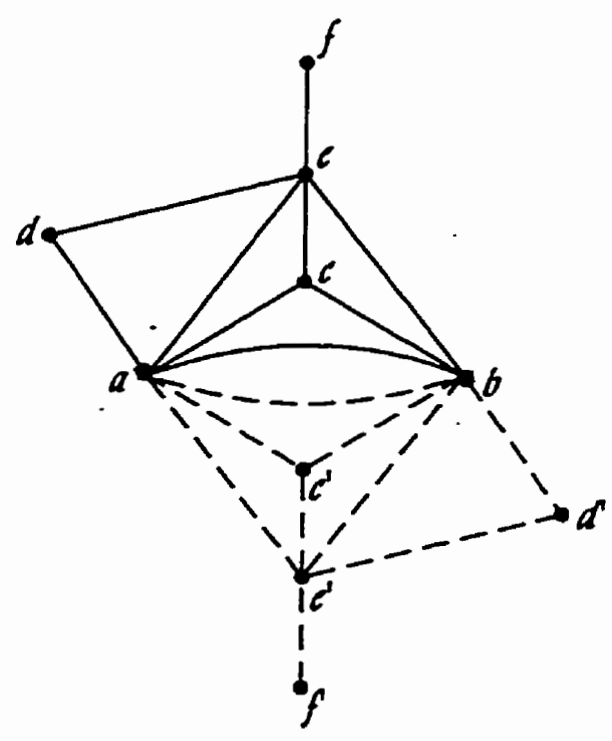

Figure 3.28: A counter-example to the question. 


\section{Bibliography}

[1] K. Appel and W. Haken. Every panar map is 4-colorable-1: Discharging. Ill.J.Math, 21:429-490, 1977.

[2] K. Appel and W. Haken. Every panar map is 4-colorable-2: Reducibility. Ill.J.Math, 21:491$567,1977$.

[3] C. Berge and V. Chvátal, editors. Topics on perfect graphs. North-Holland, Amsterdam, 1984.

[4] V. Chvátal and P.L. Hammer. Aggregation of inequality in integer programming. Annals of Disc. Math., 1:145-162, 1977.

[5] V. Chvátal, C.T. Hoàng, N.V.R. Mahadev, and D. de Werra. Four classes of perfectly orderable graphs. J. Graph Theory, 11(4):481-495, 1987.

[6] D.J.A.Welsh and M.B.Powell. An upper bound on the chromatic number of a graph and its application to timetabling problems. Computer $J_{.,}$10:85-87, 1967.

[7] M. Garey and D.S. Johnson. Computer and Intractabiliy : A Guide to the Theory of NPcompleteness. W.H. Freeman, San Fransisco, 1979.

[8] A. Ghouila-Houri. Caractérisation des graphes non orientés dont on peut orienter les arètes de manière à obtenir le graphe d'une relation d'ordre. C.R. Acad. Sci. Paris, 254:1370-1371, 1962.

[9] M.C. Golumbic. Algorithmic graph theory and perfect graphs. Academic Press, New York, 1980.

[10] P. L. Hammer and N. V. R. Mahadev. Bithreshold graphs. SIAM J. Appl. Math., pages 497-506, 1985.

[11] A. Hertz and D. de Werra. Les graphes bipolarisables. Report ORWP, Swiss Federal Institute of Technology in Lausanne, 1986.

[12] C.T. Hoàng. A note on perfectly orderable graphs. Discrete Applied Math., 1996. 
[13] C.T. Hoàng. On the complexity of recognizing a class of perfectly orderable graphs. Discret Applied Math., pages 219-226, 1996.

[14] C.T. Hoàng and N. Khouzam. On brittle graphs. J. Graph Theory, 12(3):391-404, 1988.

[15] C.T. Hoàng and B.A. Reed. Some classes of perfectly orderable graphs. J. Graph Theory. $13(4): 445-463,1989$.

[16] C. Lund and M. Yannakakis. On the hardness of approximating minimization problems. In Symposium on the Theory of Computing, pages 286-293. 1993.

[17] M. Middendorf and F. Pfeiffer. On the complexity of recognizing perfectly orderable graphs. Discrete Math., 80(3):327-333, 1990.

[18] Brooks R.L. On coloring the nodes of a network. Proc. Cambridge Phil. Soc., pages 194-197. 1941.

[19] Maciej M Syslo, Narsingh Deo, and Janusz S. Kowalik. Discrete Optimization Algorithms. Prentice-Hall, Inc., Englewood Cliffs, New Jersey 07632, 1983. 
IMAGE EVALUATION

TEST TARGET (QA-3)
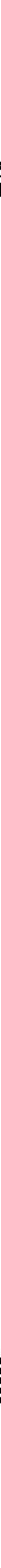